\title{
Temperature-Dependent Ellipsometry Measurements of Partial Coulomb Energy in Superconducting Cuprates
}

\author{
J. Levallois, ${ }^{1}$ M. K. Tran, ${ }^{1}$ D. Pouliot, ${ }^{2}$ C. N. Presura, ${ }^{3}$ L. H. Greene, ${ }^{2}$ J. N. Eckstein, ${ }^{2}$ J. Uccelli, ${ }^{1}$ E. Giannini, ${ }^{1}$ \\ G. D. Gu, ${ }^{4}$ A. J. Leggett, ${ }^{5, *}$ and D. van der Marel ${ }^{1, \dagger}$ \\ ${ }^{1}$ Department of Quantum Matter Physics, University of Geneva, \\ Quai Ernest-Ansermet 24, CH-1211 Genève 4, Switzerland \\ ${ }^{2}$ Department of Physics, University of Illinois at Urbana-Champaign, \\ 1110 West Green Street, Urbana, Illinois 61801, USA \\ ${ }^{3}$ Philips Research, Professor Holstlaan 4, 5656 AE Eindhoven, The Netherlands \\ ${ }^{4}$ Condensed Matter Physics and Materials Science Department, Brookhaven National Laboratory, \\ Upton, New York 11973 5000, USA \\ ${ }^{5}$ Department of Physics, University of Illinois at Urbana-Champaign, 1110 West Green Street, Urbana, \\ Illinois 61801, USA and Institute of Quantum Computing, University of Waterloo, \\ Waterloo, Ontario N2L 3G1, Canada \\ (Received 28 November 2015; revised manuscript received 24 June 2016; published 24 August 2016)
}

We performed an experimental study of the temperature and doping dependence of the energy-loss function of the bilayer and trilayer bismuth cuprates family. The primary aim is to obtain information on the energy stored in the Coulomb interaction between the conduction electrons, on the temperature dependence thereof, and on the change of Coulomb interaction when Cooper pairs are formed. We performed temperature-dependent ellipsometry measurements on several $\mathrm{Bi}_{2} \mathrm{Sr}_{2} \mathrm{CaCu}_{2} \mathrm{O}_{8-x}$ single crystals: underdoped with $T_{c}=60,70$, and $83 \mathrm{~K}$; optimally doped with $T_{c}=91 \mathrm{~K}$; overdoped with $T_{c}=84,81,70$, and $58 \mathrm{~K}$; as well as optimally doped $\mathrm{Bi}_{2} \mathrm{Sr}_{2} \mathrm{Ca}_{2} \mathrm{Cu}_{3} \mathrm{O}_{10+x}$ with $T_{c}=110 \mathrm{~K}$. Our first observation is that, as the temperature drops through $T_{c}$, the loss function in the range up to $2 \mathrm{eV}$ displays a change of temperature dependence as compared to the temperature dependence in the normal state. This effect at - or close to- $T_{c}$ depends strongly on doping, with a sign change for weak overdoping. The size of the observed change in Coulomb energy, using an extrapolation with reasonable assumptions about its $q$ dependence, is about the same size as the condensation energy that has been measured in these compounds. Our results therefore lend support to the notion that the Coulomb energy is an important factor for stabilizing the superconducting phase. Because of the restriction to small momentum, our observations do not exclude a possible significant contribution to the condensation energy of the Coulomb energy associated with the region of $q$ around $(\pi, \pi)$.

DOI: 10.1103/PhysRevX.6.031027

Subject Areas: Condensed Matter Physics

\section{INTRODUCTION}

Ever since the discovery of high- $T_{c}$ superconductivity in the cuprates, a large body of theoretical and experimental research has concentrated on the mechanism of superconductivity. The primary thermodynamic quantity of interest is the statistical average of the Hamiltonian, E. An isolated system (i.e., a system in which the entropy is conserved) becomes superconducting if, and only if, $E$ in the superconducting state is more favorable than $E$ of all alternative states of matter. Starting at the most basic level, the appropriate Hamiltonian for a system of electrons and

\footnotetext{
*aleggett@illinois.edu

†dirk.vandermarel@unige.ch
}

Published by the American Physical Society under the terms of the Creative Commons Attribution 3.0 License. Further distribution of this work must maintain attribution to the author(s) and the published article's title, journal citation, and DOI. nuclei consists of two terms, the kinetic energy (of nuclei and electrons) and Coulomb interaction energy (between nuclei and nuclei, nuclei and electrons, and electrons and electrons). At this basic level, it follows directly from the virial theorem [1] that the transition must involve saving the Coulomb energy; what is less obvious [2] is whether this is still true when one goes to the more phenomenological level of description standard in solid-state physics, where the relevant "Coulomb energy" is only that of the interaction between the conduction electrons. Several years ago, one of us [3-5] postulated that it is indeed the saving of the interconduction electron energy, and specifically the part associated with long wavelengths and mid-infrared frequencies, which is the main driver of the superconducting transition in the cuprates (the "MIR scenario"). Here, we employ a basic result from linear response theory, that the partial Coulomb energy associated with a given wave vector $\mathbf{q}$ is proportional to a thermally weighted integral of the electron energy loss function $L_{\mathbf{q}}(\omega)$ over all frequencies, 


$$
E_{C}^{\mathbf{q}}=\frac{\hbar}{2 \pi} \int_{0}^{\infty} L_{\mathbf{q}}(\omega)\left(1+2 n_{\omega}\right) d \omega
$$

where $n_{\omega}=1 /\left(\exp \left(\hbar \omega / k_{B} T\right)-1\right)$. For $q \sim 0$, the relevant loss function is that measured in optical ellipsometry. We present experimental loss-function spectra measured in this way for a series of high- $T_{c}$ cuprates with different carrier concentrations; from these data, we calculate the partial Coulomb energy $E_{C}^{0}(T)$ between 15 and $300 \mathrm{~K}$ in 1-K steps. The temperature dependence of $E_{C}^{0}$ and $\gamma_{C}=T^{-1} d E_{C}^{0} / d T$ reveals the evolution as a function of doping of the changes of Coulomb energy associated with pairing and with the superconducting phase transition. The setup for spectroscopic ellipsometry used in the present study has the advantage of high stability, high throughput, and dense sampling as a function of temperature. As a result, the energy loss spectra for $q \sim 0$ presented here, and in particular the observed subtle temperature dependencies, provide an important benchmark for future studies of the Coulomb energy using alternative methods such as transmission electron energy loss spectroscopy (EELS).

If the original MIR scenario is correct, then one would prima facie expect it to be reflected in a decrease, at and below the superconducting transition, of the loss function in the MIR region of the spectrum as measured in the optics. Thus, the first question [question (A)] that we address in this paper is a qualitative one. Namely, in the various regions of the phase diagram explored, does the small- $q$ MIR loss-function increase, decrease, or remain constant (relative to the extrapolated normal-state behavior; see below) at and below $T_{c}$ ? This question can be answered directly from the experimental data. Should the answer to this question for some particular value of doping turn out to be that it increases or remains constant, then the prima facie implication (though see below) would seem to be that the MIR scenario cannot explain the mechanism of superconductivity at least in this region of the phase diagram.

Our second question [question (B)], which is prima facie relevant only if (where) a decrease in the loss function is observed, is as follows: Is the decrease in the loss function that we measure quantitatively consistent with the MIR scenario, that is, the hypothesis that all or most of the superconducting condensation energy comes from the saving of Coulomb energy in the "small- $q$ " regime and the MIR frequency region? It should be strongly emphasized that an answer to this question requires not only a careful definition of the scenario (in particular, what we mean by small- $q$ ) but also a crucial assumption, namely, that the value of the loss function measured in our optical experiments, for which the "effective" $q$ is of the order of the inverse of the high-frequency penetration depth, about $0.002 \AA^{-1}$, can be extrapolated to the much larger values of $q$ (up to about $0.31 \AA^{-1}$ ), which dominate the theoretical expression for the Coulomb energy in the MIR scenario. In the normal phase, a comparison of the values of the loss function as measured in optical experiments with that measured in EELS is consistent with such an extrapolation [6-8]. Whether this remains valid for the changes observed at and below the superconducting transition is a question that needs to be addressed by future EELS experiments. It is worth mentioning in this context that, on the basis of inelastic neutron scattering data of the cuprates [9], indications have been obtained for a significant contribution to the condensation energy from $\mathbf{q} \sim(\pi, \pi)$.

At this point, it may be useful to review briefly the original motivation for the scenario. As we will see in more detail below [see Eq. (4)], the expectation value of the total Coulomb energy can be rigorously expressed as a sum (integral) of contributions from different Fourier components $E_{C}^{\mathbf{q}}$. As explained in Refs. [3,4], the starting observation is that one possible origin of the well-known dependence of the superconducting transition temperature $T_{c}$ on the number of $\mathrm{CuO}_{2}$ layers per unit cell is the effect of the Coulomb interaction between the conduction electrons in different planes. If indeed the saving of this energy is a major contribution to the increase of $T_{c}$ (and thus, by inference, to that of the condensation energy per plane), then since the relevant matrix element falls off as a function of the in-plane wave vector $q$ as $\exp (-q d)$, where $d=$ $3.2 \AA$ is the interplane spacing (within the bilayers), it follows that a major contribution to the saving must come from wave vectors $q<q_{0}=d^{-1}=0.31 \AA^{-1}$. It is then highly plausible (though, of course, not a rigorous statement) that the same must be true also for the Coulomb energy, i.e., that a major contribution to the condensation energy comes from the intraplane Coulomb energy with $q<q_{0}$, and we use this condition on $q$ as one of the defining ingredients in the "MIR scenario."

In Fig. 1, the partial Coulomb energy difference $\Delta E_{C}^{\mathbf{q}}$ is displayed, where $\Delta$ signifies the value of the superconducting

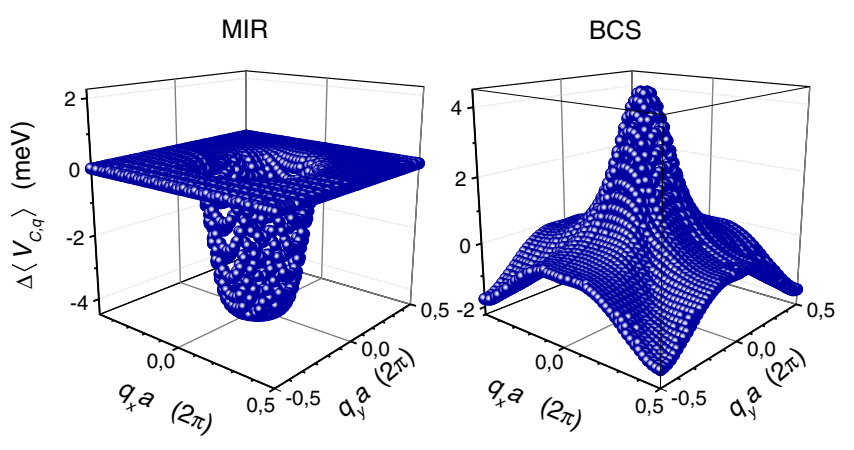

FIG. 1. S-N difference of the partial Coulomb energy $E_{\mathbf{q}}^{C}$. Left panel: According to the MIR scenario (schematic). Right panel: Resulting from a BCS model calculation [15] for $d$-wave symmetry, $p=0.16$ hole doping, and the interaction adjusted such as to give $T_{c}=100 \mathrm{~K}$. Both panels represent the $q_{z}=0$ cut in momentum space, corresponding to the electric field polarized along the planes. $E_{\mathbf{q}}^{C}$ at $\mathbf{q}=0$ corresponds to the integral [Eq. (1)] of the optical in-plane loss function. 
phase minus the one of the normal phase (hereafter called "S-N" difference for brevity). The result anticipated from the MIR scenario (left diagram) is compared with the behavior expected from BCS theory and later extensions thereof addressing the collective response [10-14] (right diagram). The BCS result [15] is a smooth function of $\mathbf{q}$ with a maximum at $q=0$. This example also demonstrates that, in the case of $d$-wave pairing, BCS theory predicts a negative sign for $\Delta E_{C}^{\mathbf{q}}$ near $(\pi, \pi)$, a state of affairs that is held responsible for stabilizing the superconducting state in the context of $t J$ and Hubbard models for high $T_{c}$ [9,16-20]. Note that, at the end of the day, this also represents a form of Coulomb energy, which is unfortunately not accessible with optical spectroscopy. The attentive reader may object that when the numbers are put in, the BCS value shows substantial variation with $q$ on the scale of the coherence length, i.e., between the optical regime and $0.31 \AA^{-1}$, whereas we are assuming the absence of such variation. There is no contradiction here: In BCS theory, the relevant frequencies that contribute are of the order of (the Fermi velocity $v_{F}$ times) $q$ itself, whereas in the MIR scenario, they are substantially larger than $v_{F} q$ even for $q \sim 0.3 \AA^{-1}$. There is no reason why the $q$ dependence should be the same in these two very different cases.

A second question relates to the region of frequency $\omega$ in which the saving occurs: It was argued in Ref. [4], following the "Willie Sutton principle," that since the only frequency regime in which the loss function in the normal state is both substantial and likely to have contributions principally from the conduction electrons in the $\mathrm{CuO}_{2}$ planes is the MIR, this is the region where the maximal saving should occur. Again, the lower and upper frequency cutoffs are somewhat arbitrary, but a natural definition of the relevant "MIR frequency regime" might be, say, $0.6-1.8 \mathrm{eV}$. In summary, the "canonical" definition of the original MIR hypothesis is that a very substantial contribution (let us say $>70 \%-80 \%$ of the whole) to the total condensation energy is made by a saving of the Coulomb energy associated with wave vectors $q<0.31 \AA^{-1}$ and with frequencies $0.6-1.8 \mathrm{eV}$, and this constitutes the conjecture addressed by our question (B). We should further note that in the original formulation, the saving was assumed to occur only at and below the macroscopic transition temperature $T_{c}$.

The above discussion obviously raises a number of further questions, which go beyond the original scenario. First, what if we relax the constraint $q<q_{0}$, i.e., consider the saving of interconduction Coulomb energy from all $q$ in the first Brillouin zone: Is it enough to constitute the whole of the condensation energy? This is an interesting question, but in order to obtain any information on it from the optical data, we would need to extrapolate the optically measured dielectric constant to the whole of the zone; this seems implausible, so we will not discuss it further here. A second generalization would be to raise the same question with the original constraint on $q$ but with the frequency regime extended to lower and/or higher frequencies (perhaps right up to the $\mathrm{x}$-ray regime). This is our question (C). Finally, we could try to relax the constraint on the relevant temperature regime and consider a generalized scenario [21] in which all or a substantial part of the energy saving takes place above the macroscopic transition; this is question (D). In the following, we will attempt to give a definitive answer to question (A), a relatively definitive one (subject to the extrapolation assumption) to question (B), and some information that, while it does not answer questions (C) or (D) unambiguously, may be qualitatively relevant to them.

\section{COULOMB ENERGY IN SUPERCONDUCTORS}

\section{A. Sum rules}

To motivate this subsection, we briefly recapitulate the fundamental concepts underlying the MIR scenario. The theoretical description of the conduction electrons in the cuprates is based on the following key assumptions [3]: (i) Core and conduction electrons can be treated as separate systems. (ii) The loss spectra below $2 \mathrm{eV}$ are dominated by the $\mathrm{CuO}_{2}$ planes. (iii) Ionic motion (phonons) is irrelevant. (iv) The optical response and the mechanism of pairing are essentially two dimensional; i.e., it is justified to neglect intermultilayer tunneling in the analysis of superconductivity (details are provided in Appendix G). Thus, the generic Hamiltonian is written as

$$
\hat{H}=\hat{T}+\hat{U}+\hat{V}_{C},
$$

with $\hat{T}$ the in-plane kinetic energy, $\hat{U}$ the potential felt by the electrons due to the ionic cores, and $\hat{V}_{C}$ the conduction electron-electron Coulomb interaction energy [22]. The main postulate of the MIR scenario is that the interaction energy $\left\langle\hat{V}_{C}\right\rangle$ decreases upon entering the superconducting state.

The first purpose of the present paper is to explore the qualitative consistency of the optical data with the MIR scenario, i.e., to answer question (A). For this limited purpose, we ignore complications associated both with the layered nature of the cuprates (i.e., we treat them for electrodynamic purposes as 3D continua) and with the screening of the Coulomb interaction by the ionic cores (for these complications, see Ref. [7] and Appendixes F and G). The total energy per unit cell contained in the interparticle Coulomb energy is provided by the relation

$$
E_{C}=\left\langle\hat{V}_{C}\right\rangle=\frac{1}{2 N} \sum_{\mathbf{q}} V_{\mathbf{q}} S_{\mathbf{q}}
$$

where $N$ is the number of unit cells, $S_{\mathbf{q}}=\left\langle\hat{\rho}_{-\mathbf{q}} \hat{\rho}_{\mathbf{q}}\right\rangle$ the structure factor, and $V_{\mathbf{q}}=4 \pi e^{2} / q^{2}$ the Fourier transform 
of the Coulomb potential. For the expression on the righthand side, we can employ the general relation [23] between the structure factor and the charge susceptibility following from the fluctuation-dissipation theorem

$$
S_{\mathbf{q}}=\frac{1}{\pi} \int_{0}^{\infty} \chi^{\prime \prime}(\mathbf{q}, \omega)\left(1+2 n_{\omega}\right) \hbar d \omega .
$$

The susceptibility appearing in this expression, $\chi(\mathbf{q}, \omega)$, measures the charge response to a density perturbation with frequency $\omega$ and wave vector $\mathbf{q}$, and is related to the longitudinal dielectric function $\epsilon_{\|}(\mathbf{q}, \omega)$ through

$$
\frac{1}{\epsilon_{\|}(\mathbf{q}, \omega)}=1-V_{q} \chi(\mathbf{q}, \omega) .
$$

The imaginary part of Eq. (5),

$$
L_{\mathbf{q}}(\omega)=\operatorname{Im} \frac{-1}{\epsilon_{\|}(\mathbf{q}, \omega)},
$$

can be measured with the help of inelastic electron scattering [6] and is, for this reason, called the electron energy loss function. The corresponding transverse quantity, in which $\epsilon_{\|}$is replaced by $\epsilon_{\perp}$, can be measured in the $q \rightarrow 0$ limit by optical spectroscopy. For normal metals, it is well established that, in the limit of $q \rightarrow 0$, one has $\varepsilon_{\|}(\mathbf{q}, \omega)=\varepsilon_{\perp}(\mathbf{q}, \omega)$. This has recently been proven also for the superconducting state by two of us [21] in the relevant limit $q \rightarrow 0, \omega \neq 0$. The equivalence in the case of the cuprates is, for example, illustrated in Fig. 3 of Ref. [8]. Together, Eqs. (3), (4), (5), and (6) provide the "Coulomb energy sum rule" [3-5,23-25], which, generalized to finite temperature, provides the Coulomb interaction energy

$$
E_{C}=\frac{1}{N} \sum_{\mathbf{q}} E_{C}^{\mathbf{q}}
$$

where $E_{C}^{\mathbf{q}}$ is given by Eq. (1).

It should be noted that neither the form of the Hamiltonian (2) nor the above derivation of the Coulomb energy sum rule necessarily implies that the standard textbook description of the many-body conduction-electron wave function as an approximately Slater determinant of Bloch waves is a good one. However, we can always use the Bloch waves as a basis, and if we do so, then one consequence of the occurrence of the periodic crystalline potential $U$ in the Hamiltonian (2) is the occurrence of "Umklapp" scattering processes. In a recent study, Lee calculated the influence of Umklapp processes on the spectral weight of the loss function near the plasma resonance and predicted an increase of plasmon spectral weight as the system undergoes the superconducting phase transition [14].
For the special limit in which, in the band picture [i.e., the set of energy eigenstates of the single-particle terms $\hat{T}+\hat{U}$ in Eq. (2)], the lowest relevant band reduces to a nearest-neighbor tight-binding model and one assumes that the interacting conduction electrons are confined to this band, there exists a second well-known sum rule for the "kinetic" energy per unit cell [26-29]:

$$
K=-\frac{\hbar^{2} \Omega_{0}}{\pi^{2} e^{2} a^{2}} \int_{0}^{\infty} \omega \operatorname{Im} \epsilon(\omega) d \omega,
$$

where $a$ is the in-plane lattice parameter and $\Omega_{0}$ is the unit cell volume. $K$ subsumes the contributions from the first two terms of Eq. (2), in addition to Hartree-Fock (and higher-order) contributions from the interaction term. Equation (8) is therefore, to some extent, complementary to the Coulomb-energy sum rule, Eq. (7). Equation (8) has been the subject of intensive investigations pertaining to the question of whether superconductivity in the cuprates is caused by a lowering of kinetic (i.e., single-particle) energy $[2,30,31]$. Interestingly, it turns out that in underdoped samples of the cuprates, the kinetic energy behaves oppositely to the BCS prediction (i.e., is decreased by the $\mathrm{N}-\mathrm{S}$ transition), while on the overdoped side, it behaves consistently with BCS (i.e., is increased) [32,33], which is in fact consistent with numerical calculations based on the Hubbard model and the $t-J$ model [18-20]. However, one should beware of assuming that the kinetic (single-particle) energy that enters the sum rule (8) is necessarily the expectation value of the sum of the single-particle terms $\hat{T}$ and $\hat{U}$ in Eq. (2); the tight-binding description leading to Eq. (8) is at a different level from that of the Hamiltonian (2), and it is, for example, not excluded that the Coulomb term in Eq. (2) may affect the effective tunnelling matrix elements in the tight-binding description. Thus, should it, for example, be found experimentally that in some doping intervals both the rhs of Eq. (7) and the rhs of Eq. (8) decrease at the $\mathrm{N}-\mathrm{S}$ transition, this would not necessarily constitute a paradox.

\section{B. Optical data and the MIR scenario}

As mentioned, the present work aims at exploring the energy stored in the inter-electronic Coulomb interactions, using precise measurements and analysis of the optical loss function. As emphasized in the Introduction, to infer anything about the Coulomb energy from the optical data, we need to extrapolate our results to finite $q$. The big advantage of optics is the possibility to acquire data during extended periods of time. This allows us to obtain detailed information about the relative changes of Coulomb energy as a function of temperature and doping. These results in turn provide a benchmark for the accuracy needed to detect these trends with momentum-sensitive techniques such as inelastic neutron scattering, (resonant) inelastic x-ray scattering, or electron energy loss spectroscopy, which are 
subject to the severe constraints on measurement time inherent to large facilities.

\section{METHODS}

\section{A. Samples}

We investigated high-purity single crystals of $\mathrm{Bi}_{2} \mathrm{Sr}_{2} \mathrm{Ca}_{2} \mathrm{Cu}_{3} \mathrm{O}_{10+x}$ (Bi2223) and of $\mathrm{Bi}_{2} \mathrm{Sr}_{2} \mathrm{CaCu}_{2} \mathrm{O}_{8-x}$ (Bi2212), with $a b$-plane oriented surfaces of several $\mathrm{mm}^{2}$. The Bi2212 samples are easily cleavable, providing clean and mirrorlike surfaces for optical studies. Details on growth and characterization of the crystals are provided in Appendix A. We use the empirical Tallon-Presland relation between carrier concentration and $T_{c}[34,35]$ to determine the doping $p$. For our under and optimally doped Bi2212 samples with $T_{c}=60,70,83$, and $91 \mathrm{~K}$; this yields $p=0.1,0.11,0.13$, and 0.16 , respectively. For the overdoped Bi2212 samples with $T_{c}=84,81,70$, and $58 \mathrm{~K}$, we get $p=0.19,0.2,0.21$, and 0.23 , respectively.

\section{B. Ellipsometry measurements}

Using ellipsometry at an angle of incidence of $70^{\circ}$ with the surface normal, we measured the real and imaginary parts of the ratio of $p$-polarized over $s$-polarized complex reflectivity coefficients, $\rho=r_{p} / r_{s}$ (see Appendix B). The spectrum is measured continuously while the temperature is varied from $15 \mathrm{~K}$ to $300 \mathrm{~K}$ at a rate of $0.2 \mathrm{~K} / \mathrm{min}$. For an isotropic material, the dielectric function $\epsilon(\omega, T)$ can be readily obtained by numerical evaluation of the relation

$$
\epsilon(\omega, T)=\sin ^{2} \theta+\frac{\sin ^{4} \theta}{\cos ^{2} \theta}\left(\frac{1-\rho}{1+\rho}\right)^{2}
$$

following from the Fresnel equations, where $\theta$ is the angle of incidence, and the loss function is

$$
L(\omega, T)=\operatorname{Im} \frac{-1}{\epsilon(\omega, T)} .
$$

For an optically anisotropic material, the expressions are more complicated, but often, as in the present case, the $c$-axis admixture is small and can be corrected easily using a rapidly converging iterative method, which is outlined in Appendix D. The loss function yields, through frequency integration, the partial Coulomb energy, Eq. (1). For brevity, we will drop the momentum $\mathbf{q}$ in the subsequent discussion of the optical properties, with the understanding that the experimental data presented here are representative of $q \sim 0$. For the purposes of the present study, it is convenient to also define an integral between (sampledependent) limits $\omega_{1}, \omega_{2}$, which are chosen so that the integration runs over a frequency interval where the loss function has the same sign of temperature variation (in our case, it increases upon cooling); i.e., $\omega_{1}$ and $\omega_{2}$ are, to a very good approximation, isosbestic points [this is illustrated in Fig. 19 (Appendix E) by the enlarged view of one of the samples, which is indeed representative of all samples studied here]. This implies that the slope of the intensity versus temperature is opposite in the region between the isosbestic points and the frequency regions below and above. While this applies to the general trend over the full 300-K span of temperatures, it does not apply to the sudden change of slope at $T_{c}$. In the examples that follow, we will see that change of slope is either much smaller-but with the same sign-below and above the interisosbestic region, or zero within the experimental accuracy. The intraisosbestic integral

$$
E_{C}^{\mathrm{iso}}(T)=\frac{\hbar}{2 \pi} \int_{\omega_{1}}^{\omega_{2}} L(\omega, T)\left(1+2 n_{\omega}\right) d \omega
$$

eliminates compensation of opposite temperature trends in different parts of the spectra and provides, for this reason, the cleanest (i.e., noise-free) representation of the temperature dependence of the experimental spectra in the region of the maximum of the loss function. This is also the spectral range where, faithful to the "Willie Sutton principle" [36] we anticipate the strongest saving of Coulomb energy. At the same time, since the definition of Coulomb energy requires integrating over all energy, it is also interesting to look in the other parts of the spectrum. To address these contributions to the Coulomb energy, the temperature dependence of the loss-function integral below the lower isosbestic point and above the upper isosbestic points is reported for each sample in Appendix C.

To motivate the form in which we present our data, we draw an analogy between $E_{C}(T)$ and the total internal energy $E(T)$. In general, for a system in thermal equilibrium, $E(T)$ is an increasing function of temperature, with a discontinuity in the slope at a second-order transition. Usually, one does not measure the internal energy itself but rather its temperature derivative $C_{V}=d E / d T$, i.e., the specific heat, which has a jump at a second-order phase transition. Often, in the cuprates, the specific heat presents a $\Lambda$-like transition at $T_{c}$ rather than a jump. This type of broadening is often attributed to superconducting fluctuations, which must be properly accounted for when one tries to extract the condensation energy $E_{\text {cond }}$ from the data [37]. Since the specific heat of a metal in the normal state is characterized by a linear temperature dependence ( $E$ being proportional to $T^{2}$ as discussed in Appendix $\mathrm{H}$ ), it is common practice to display the Sommerfeld coefficient $\gamma=T^{-1} C_{V}(T)$. In the present context, we concentrate our analysis on the corresponding quantity related to the Coulomb energy,

$$
\gamma_{C}^{\text {iso }}(T)=\frac{1}{T} \frac{d E_{C}^{\text {iso }}(T)}{d T}
$$


Since numerical evaluation of the temperature derivatives causes a strong amplification of the experimental noise, we can-for this purpose-only use $E_{C}^{\text {iso }}(T)$, i.e., the loss function integrated between the two (sample-dependent) isosbestic points.

\section{LOSS FUNCTION AND PARTIAL COULOMB ENERGY CHANGE THROUGH THE SUPERCONDUCTING PHASE TRANSITION}

\section{A. Qualitative features}

The loss function for the temperatures ranging from 15 to $300 \mathrm{~K}$ for all Bi2212 and Bi2223 samples is displayed in Figs. 2 and 3. The peak of the loss function corresponds to the plasma-resonance energy, which is at an energy slightly above $1 \mathrm{eV}$ in all samples. Common to all samples of this study, the intensity in the energy loss function increases gradually when cooling down and gains approximately 5\% between $300 \mathrm{~K}$ and $15 \mathrm{~K}$. There is a narrowing of the lossfunction peak and a blueshift of about 5\%. For each of the samples, all curves measured at different temperatures cross at two isosbestic points on either side of the maximum of the loss function.

The real and imaginary parts of $-\epsilon(\omega)^{-1}$ for all samples are shown for different temperatures in Figs. 8-15 of Appendix C. In the same figures, we also compare the temperature dependence of the intraisosbestic loss-function intensity $E_{C}^{\text {iso }}(T)$, and the loss function integrated from 0 to $2.5 \mathrm{eV}$ (right panels of the second and third rows). In the second (third) line of the first column, we display the temperature dependence of the loss-function integrals in the range below (above) the intraisosbestic region. For ease of comparison of the contributions from the different energy ranges, these contributions are indicated on the same scale for a given sample.

Based on these data, we make the following global observations: In the first place, the loss-function integrals from 0 to $\hbar \omega_{1}$ (second row, left panel) and $\hbar \omega_{2}$ to $2.5 \mathrm{eV}$ (third row, left panel) have very weak temperature dependence compared to the intraisosbestic loss-function integrals (from $\hbar \omega_{1}$ to $\hbar \omega_{2}$ ). In the second place, the loss-function integrals over the full $(0$ to $2.5 \mathrm{eV})$ range (third row, right
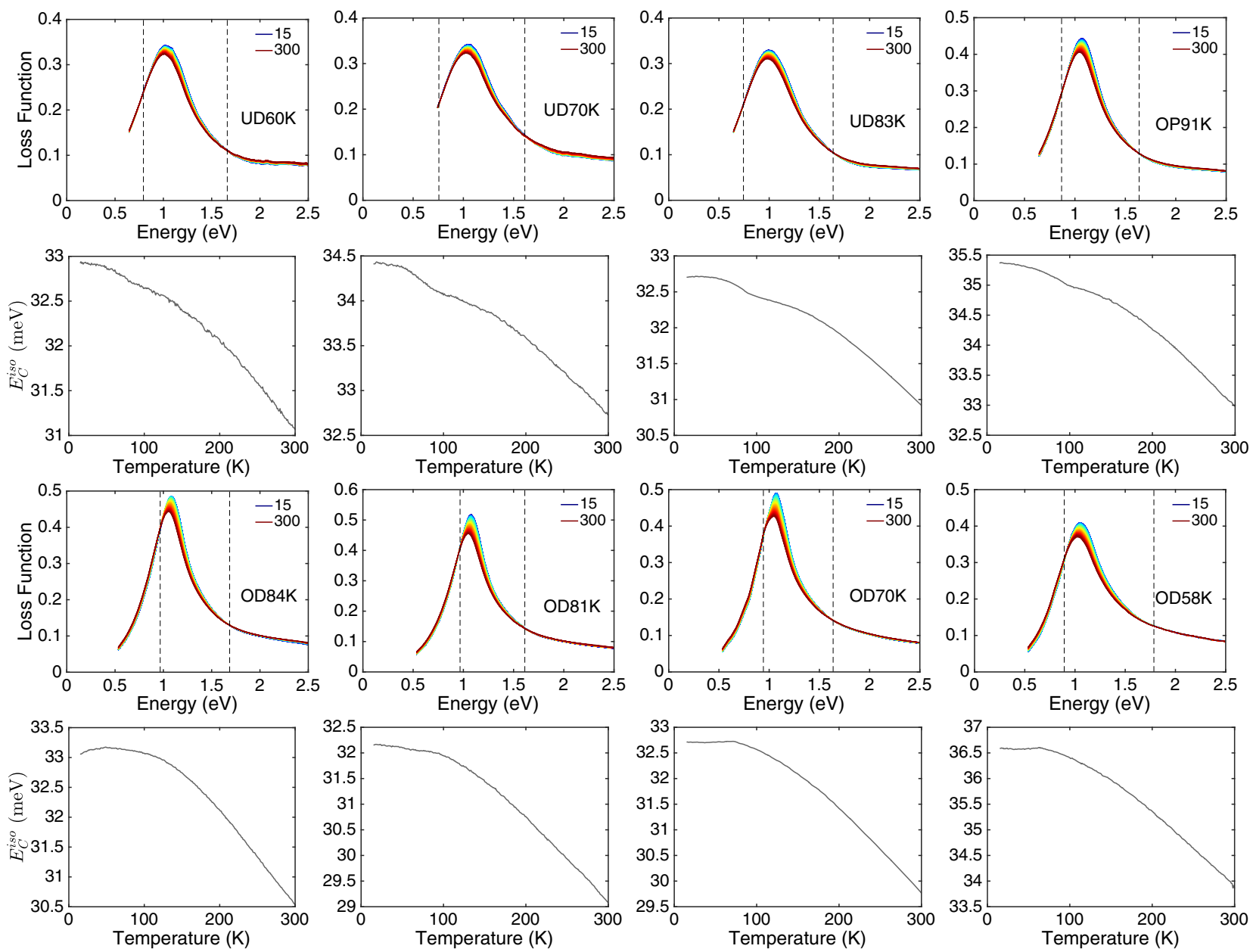

FIG. 2. Loss-function spectra for Bi2212 with carrier concentrations ranging from underdoped to overdoped for selected temperatures (lines 1 and 3). Temperature dependence of the integrated intensity, $E_{C}^{\text {iso }}(T)$, of the corresponding samples (lines 2 and 4). 

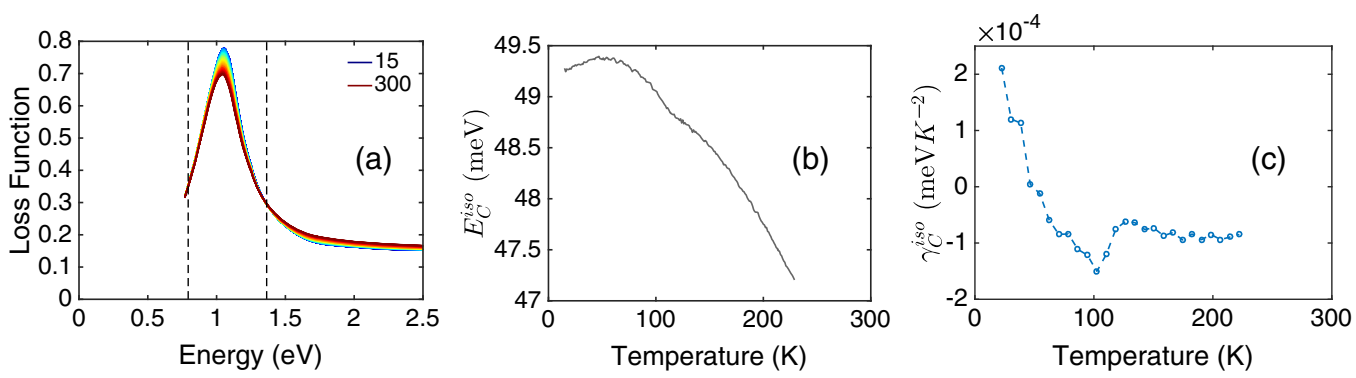

FIG. 3. Optimally doped Bi2223. (a) Loss-function spectra, (b) temperature dependence of the integrated intensity $E_{C}^{\text {iso }}(T)$, and (c) temperature dependence of $\gamma_{C}^{\text {iso }}(T)$.

panels) show, by and large, the same main features as the intraisosbestic loss-function integrals while exhibiting stronger experimental noise. In view of these observations, and in the interest of the best possible signal-to-noise ratio, for the details of the temperature dependence, we concentrate on the intraisosbestic loss-function integrals $E_{C}^{\text {iso }}(T)$, which are displayed for all samples in the second and fourth lines of Fig. 2. However, it is important to emphasize that this choice in no way influences our conclusions about the temperature dependence through $T_{c}$ : The extended integrals from 0 to $2.5 \mathrm{eV}$, displayed in Figs. 8-15 of Appendix C, show, for all samples, the same effects, both qualitatively and quantitatively, when $T$ is tuned through the superconducting phase transition. The key aspects of the observed temperature dependencies and the evolution thereof as a function of doping are the following:

(1) In the underdoped samples, we observe in $E_{C}^{\text {iso }}(T)$ an upward kink at $T_{n}$. An upward kink is also observed at $T_{n}$ for both optimally doped bilayer Bi2212 and for the trilayer compound Bi2223.

(2) In the overdoped samples, $E_{C}^{\text {iso }}(T)$ shows a downward kink below a temperature $T_{p}$.

(3) For $\operatorname{Bi2223,} E_{C}^{\text {iso }}(T)$ turns downward below $60 \mathrm{~K}$. We speculate that this behavior has to do with the peculiarity that the two outer planes and the inner plane of the trilayer compound have very different doping levels, as has been noticed with nuclear magnetic resonance [38,39] and angle-resolved photoemission (ARPES) [40]. Based on an analysis of the ARPES data, it was estimated [40] that the outer planes are overdoped with $x=0.23$ holes per copper atom, while the inner plane is strongly underdoped with only $x=0.07$ holes per copper. This corresponds to an average doping $x=0.18$. In this case, we expect a rich temperature dependence of $E_{C}^{\text {iso }}(T)$ of the coupled planes, combining aspects of both the underdoped and the overdoped sides of the phase diagram, which indeed appears to be the case for the Bi2223 data (see Fig. 3). Figure 4 displays $\gamma_{C}^{\text {iso }}(T)$ evaluated using Eq. (12). The main feature revealed by this quantity is the extremum close to $T_{c}$. Note the striking similarity of $\gamma_{C}^{\text {iso }}(T)$ in the overdoped samples to the Sommerfeld coefficient measured in a canonical superconductor. At the underdoped side, the transition is broader, and $\gamma_{C}^{\text {iso }}(T)$ has a $\Lambda$-like appearance, similar to the Sommerfeld coefficient of underdoped cuprates obtained from specific heat experiments [37, 41-43]. Of particular interest is the opposite sign of this extremum when comparing the underdoped and overdoped samples. This sign change of the jump of $\gamma_{C}^{\text {iso }}(T)$ occurs for $p \approx 0.19$, which coincides with the point where a large body of experimental data indicates the closing of the pseudogap [43]. Recently, Lee [14] has obtained, from a formalism taking into account Umklapp processes, that the spectral weight in the loss-function peak is enhanced when the material switches from normal to superconducting. While this prediction agrees with the behavior that we observe for the underdoped samples, it is opposite to the effects seen on the overdoped side. Further theoretical studies should clarify the role and impact of the Umklapp processes as a function of doping.

In any case, the answer to question (A) (given, as always, the extrapolation assumption) is clear: The optical data are qualitatively consistent with the MIR scenario on the overdoped side of the phase diagram but not on the underdoped side.

\section{B. Quantitative considerations}

We now turn to question (B). To quantify the change of Coulomb energy in the $\mathrm{N}-\mathrm{S}$ phase transition, we proceed in two steps: (i) We need to compare the measured data of the samples, which are superconducting, to the value without superconductivity. (ii) We need to estimate the average over the relevant sector of $\mathbf{q}$-space actually not of the loss function itself but of a related quantity (see below). For point (i), we essentially need to extrapolate the normal state trend to zero. For the optimally doped sample with $T_{c}=91 \mathrm{~K}$, the integrated loss function has a clean kink at $T_{c}$ (Fig. 2, top right panel), and the behavior below $T_{c}$ behaves as $E_{C, s}^{0}(T)=E_{C, n}^{0}(T)+\Delta E_{C}^{0}\left[1-\left(T / T_{c}\right)^{\eta}\right]$, with $\Delta E_{C}^{0}=0.2 \mathrm{meV}$ and $1.5 \lesssim \eta \lesssim 3$. The constant $\Delta E_{C}^{0}$ then 

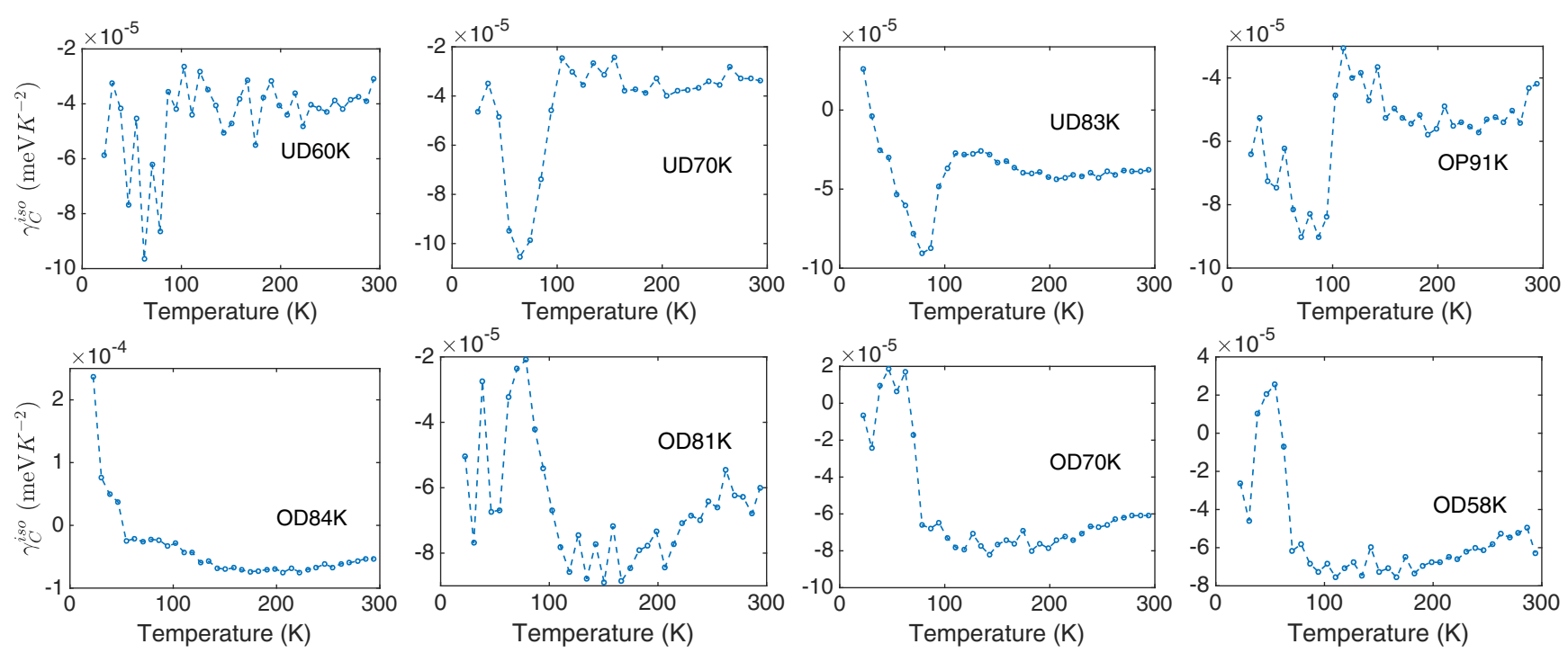

FIG. 4. Temperature dependence of $\gamma_{C}^{\text {iso }}(T)$ for Bi2212 with different carrier concentrations.

represents the S-N difference of partial Coulomb energy for $q \sim 0$. For some of the samples, the transition is less sharp, the normal state trend is less obvious, or a combination of these. To assure that the results of different dopings can be compared to each other, we calculate $\Delta E_{C}$ for each of the samples using the expression

$$
\Delta E_{C}^{0}=\eta^{-1}\left[\gamma_{C}\left(T_{2}\right)-\gamma_{C}\left(T_{1}\right)\right] T_{c}^{2},
$$

where $T_{1} \sim T_{c}$ and $T_{2}$ are the temperatures shown in Fig. 6 characterizing the step in $\gamma_{C}(T)$. Since the choice $\eta=2$ provides, for sample Op91, the expected result $\Delta E_{C}^{0} \sim 0.2 \mathrm{meV}$, we use $\eta=2$ for all samples. The quantity $\Delta E_{C}^{0}$ forms a useful standard of comparison for the energies to be discussed below.

Point (ii) involves some rather delicate considerations concerning the meaning of the "MIR scenario." To motivate them, let us note that the total Coulomb energy associated with wave vector $\mathbf{q}$ is given rigorously by Eq. (1). Thus, if we make our extrapolation assumption and assume for the moment that $L$ is not strongly dependent on the $c$-axis component of $\mathbf{q}$, the order of magnitude of the contribution to the total Coulomb energy from "small $q$ and mid-infrared $\omega$ " is simply given by $\Delta E_{C}^{0}$ multiplied by the fraction of the first Brillouin zone corresponding to the in-plane component of $\mathbf{q}$ being less than $q_{0}=0.31 \AA^{-1}$. This fraction is about $10 \%$, so the resulting energy is about an order of magnitude smaller than the experimentally measured condensation energy. However, this estimate is not in the spirit of the MIR scenario, which attributes the condensation energy to the saving of the Coulomb interaction energy between the conduction electrons in the $\mathrm{CuO}_{2}$ planes; note that this interaction is screened by the core electrons, an effect that turns out to be quite significant quantitatively.
We should therefore calculate this interaction energy (or rather the S-N difference in it) along the lines of Refs. [3,4] or via a related "3D" approach; see Appendix G. To object that while the fraction of $\Delta E_{C}^{0}$ thus obtained may be quite large, the total Coulomb energy saving associated with small $q$ and mid-infrared $\omega$ is much smaller, is no more compelling than would be an objection to the physical relevance of the "kinetic energy sum rule" (cf. Sec. II) on the grounds that it does not take account of the change of kinetic energy of the core electrons. In both cases, we are exploring the situation at the level of a model, and the outcome may look qualitatively different from the exact Dirac-level picture.

The quantity whose difference in the $\mathrm{N}$ and $\mathrm{S}$ phases we want to estimate is given by Eq. (5.1.1) of Ref. [4], with the factor $K(\omega)$ given by Eq. (4.1.4) of that reference; for the special case (relevant to $\mathrm{Bi}-2212$ ) of $n=2$, the resulting expression for the MIR scenario is

$$
\begin{aligned}
E_{C}^{\mathrm{mir}}= & \frac{a^{2}}{8 \pi^{2}} \int_{0}^{q_{0}} q d q \int_{0}^{\infty} d \omega \\
& \times \sum_{p= \pm 1} \operatorname{Im} \frac{-1}{1+\left[1+p e^{-q d}\right](q \bar{d} / 2)\left[\epsilon(\omega)-\epsilon_{b}\right] / \epsilon_{\mathrm{sc}}} .
\end{aligned}
$$

In expression (14), the quantity $\bar{d}$ is the mean plane spacing (7.8 $\AA$ for $\mathrm{Bi}-2212), \epsilon_{\mathrm{sc}}$ is the factor (assumed to be frequency independent) by which the Coulomb interaction between the conduction electrons in the $\mathrm{CuO}_{2}$ planes is screened by the ionic cores, and $\epsilon_{b}$ is the "background" dielectric constant, arising from not only the in-plane $\mathrm{Cu}$ and $\mathrm{O}$ ions and the intercalated $\mathrm{Ca}$ but also from the ions in the "charge-reservoir" layers, which has to be subtracted from the experimentally measured $\epsilon(\omega)$ to get the 


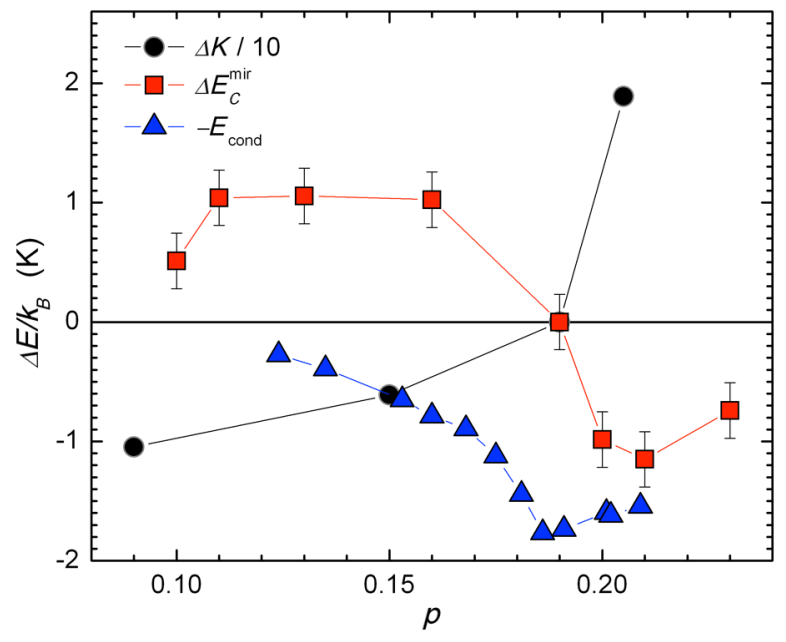

FIG. 5. The S-N difference of the q-integrated Coulomb energy $\Delta E_{C}^{\mathrm{mir}}$, together with the total energy difference $-E_{\text {cond }}$ (data reproduced from Ref. [44], with original units converted to the present ones for the sake of comparison) and band-energy difference $\Delta K[33]$.

conduction-electron contribution. In evaluating expression (14), we have taken $\epsilon_{\mathrm{sc}}=\epsilon_{b}=4.5$ (see Appendix F). Formula (14) is based on a "2D" treatment ("method 1") in which one regards the planes as separate from the 3D "background" and ignores the effect of interplanar Coulomb interactions, which are important only for $q d_{c} \lesssim 1$, i.e., $q \lesssim 0.065 \AA^{-1}$, where $d_{c}$ is the interplanar spacing. In Appendix G, we generalize the expressions to a finite interplanar Coulomb interaction. For the present parameters, the different methods, i.e., modeling as a 3D stack of $\delta$ layers [Eq. (G10)] or as a bilayer [Eq. (14)] result in the same estimate of the momentum-integrated Coulomb energy (see Fig. 22), showing that the finite $\mathbf{q}$ extrapolation effectively corresponds to multiplying the $q=0$ value by a factor $F=0.42$. Using the same method, we obtained $F$ for all other dopings, giving $F \sim 0.4$ with weak sample-tosample variations. Together with the output of Eq. (13), this yields the MIR-regime Coulomb energy $\Delta E_{C}^{\mathrm{mir}}=F \Delta E_{C}^{0}$.

The doping dependence of $\Delta E_{C}^{\mathrm{mir}}$ is presented in Fig. 5. This figure constitutes the central result of this study. In addition, we show the $\mathrm{S}-\mathrm{N}$ difference of the total energy $-E_{\text {cond }}$ (i.e., minus the condensation energy) obtained from specific heat [44], and $\Delta K$ from the sum rule, Eq. (8), representing the difference in band energy ("kinetic energy") between the superconducting and normal states [33]. Important for the interpretation of the data is the comparison of the absolute values of the energies involved: The Coulomb energy change is in the range -1 to $1 \mathrm{~K}$, the condensation energies are in the range 0 to $2 \mathrm{~K}$, and the kinetic energy changes are in the range -10 to $20 \mathrm{~K}$. We note (a) that the general trend of the MIR-regime Coulomb energy as a function of doping, in strong contrast to that of the kinetic energy, is similar to that of the total condensation energy and (b) that in the overdoped region $(p>0.19)$, it can contribute to the latter, though it obviously cannot be the whole cause of superconductivity. This answers the question stated in the Introduction of this paper, namely, whether the saving of the interconduction electron energy, and specifically the part associated with long wavelengths and mid-infrared frequencies, drives the superconducting transition in the cuprates. The answer to this question is as follows: Is it an important factor in the energy balance, but not the only factor driving the mechanism of pair formation. However, to obtain this answer we have assumed, rather than tested experimentally, the momentum dependence predicted by the MIR model. Future experimental studies using electron energy loss spectroscopy are needed to test the prediction about the momentum dependence of the partial Coulomb energy.

These results do not exclude the possibility that the Coulomb energy in the $(\pi, \pi)$ region is important for stabilizing the superconducting state. On the microscopic level, this can involve superexchange interaction mediated by the virtual exchange of spin fluctuations, or other manybody effects involving the Coulomb potential. The results in Fig. 5 show a striking similarity with the theoretical results of Gull and Millis (GM), shown in the lower panel Fig. 2 of Ref. [19]. These results were obtained using the dynamical cluster approximation (DCA) version of dynamical mean-field theory [45] for the Hubbard model, taking the value $U=6 t$ for the on-site repulsive interaction. In GM. the kinetic energy and interaction energy refer to the expectation values of the corresponding two terms (the only ones) of their Hamiltonian. Particularly striking is the agreement with the change of sign of $\Delta K$ and $\Delta E_{C}$, which follows the same trends both in experimental and computational data. The doping level where the sign change occurs is different ( $p=0.08$ in Fig. 2 of Ref. [19]), which is not surprising considering that the band-structure details are different between the theoretical model and Bi2212. Moreover, in a recent numerical study, Fratino et al. found that this crossing point depends strongly on the $U$ over bandwidth ratio [20]. The most important difference with the numbers shown in Fig. 5 is that the interaction energy in the latter refers to the long-wavelength limit of the Coulomb energy, which in reality diverges as $e^{2} / q^{2}$, whereas the Hubbard interaction is independent of $q$. It would be interesting to analyze GM's method to see how the saving of interaction energy depends on $q$ and to study extended versions of this model to include the $e^{2} / q^{2}$ dependence in the long-wavelength limit. In their numerical data, GM find no indication that the nonsuperconducting pseudogap state has any significant pairing correlations. The sign change seen both in the theoretical and in the experimental data is, however, compatible with a competition between the pseudogap, present at the underdoped side, and superconductivity. 


\section{HIGHER-FREQUENCY AND HIGHER-TEMPERATURE EFFECTS}

Because of the fact that the Coulomb energy cannot be treated perturbatively, the question as to which energy regime contributes most to the condensation energy is very difficult to answer theoretically. The emphasis on the photon energy range from 0.6 to $1.8 \mathrm{eV}$ was made primarily because this is the most obvious regime where the normal state loss function is both substantial and reasonably system independent. This assumption may have been too restrictive since even if interband transitions dominate the optical response above $2 \mathrm{eV}$, these bands are probably mostly associated with the $\mathrm{CuO}_{2}$ planes and/or the apical oxygen, which are common to the various cuprates. For example, Eqs. (14) and (G10) implicitly attribute a fraction $1-1 / \epsilon_{\mathrm{sc}}$ of $\Delta E_{C}^{\mathrm{mir}}$ reported in Fig. 5 to the range of interband transitions of the loss function (for details, see Appendix F). These considerations lead to hypothesis C: An important contribution to the Coulomb energy saving originates in the energy range of charge-transfer transitions above the free-carrier plasma frequency.

We note one further point, namely, that originally it was assumed [3] that the saving of the Coulomb energy would set in at $T_{c}$; however, more recently, and in the light of the earlier rounds of this experiment, two of us [21] have considered the possibility, in a more general scenario, of the onset of a drop in the Coulomb energy at some temperature fairly well above $T_{c}$ - crudely speaking, because of preformed Cooper pairs [46] — in a temperature range where other experiments seem consistent with the onset of local Cooper pairing [47-54]. These considerations lead to hypothesis D: The experimental results obtained up to this point could be compatible with a gradual process, whereby upon lowering the temperature, finite-range pair correlations become progressively facilitated by a saving of Coulomb energy.

\section{A. Hypothesis C: Susceptibility well above the plasma frequency}

In order to investigate the temperature dependence of the loss function well above the main plasmon peak, we analyze the Coulomb energy integrated from zero up to $2.5 \mathrm{eV}$ (in view of the large amount of noise in some of the samples in the range 2.5-3.1 eV, we omit this range from the integral). For frequencies below the frequency range of the instrument $(0.6 \mathrm{eV})$, the loss function was obtained from a Drude-Lorentz fit to the experimental data of both real and imaginary parts of $\epsilon(\omega)$ simultaneously. While this does not provide fine details such as optical phonons, the real and imaginary parts of $\epsilon(\omega)$ in the range 0.6 to $3.1 \mathrm{eV}$ narrowly constrain the range of possible values of the lossfunction integral between 0 and $0.6 \mathrm{eV}$, as revealed by the low noise level of the temperature dependence shown in the middle left panels of Figs. 8-15. In Appendix E, we demonstrate the validity of this procedure for the example of Bi2223 by comparing the extrapolation with experimental data in the low frequency range. Comparing the middle left panels with the middle right panels of all samples, we see that this energy range has a smooth and weak temperature dependence; in particular, the trends at the superconducting transition are not affected if we add this part to the integral. The energy range between the upper isosbestic point and $2.5 \mathrm{eV}$ is more affected by instrument noise. The general trend as a function of doping and temperature is that the temperature dependence increasingly approaches a $T$-linear behavior towards overdoping, and this trend is more pronounced when we extend the integration range from an upper isosbestic point to $2.5 \mathrm{eV}$.

Taking the sum of all three zones results in curves for optimally doped and weakly underdoped samples showing maxima at around $150 \mathrm{~K}$. These maxima are a direct consequence of adding the intraisosbestic $B-A T^{\alpha}$ with $\alpha \sim 2$ and the contributions from zones 1 and 3 of the form $C+D T^{\beta}$, where $\beta \sim 1$. In view of our current lack of understanding of these temperature dependences, it is unclear whether the maxima have any significance within the MIR scenario.

All in all, the verdict regarding hypothesis $\mathrm{C}$ is as follows: The energy range of charge-transfer transitions above the free-carrier plasma frequency contributes significantly to the temperature dependence of the normal state. As far as the changes across the superconducting phase transition are concerned, the contribution to the Coulomb energy in the narrow range of about $1 \mathrm{eV}$ above the plasma frequency is too small to be observable in most of our samples.

\section{B. Hypothesis D: Search for fluctuations far above $T_{c}$}

In relation to hypothesis $\mathrm{D}$, we point out recent experiments $[48,55]$ indicating that, in the cuprates, superconductivity competes with various different states of matter. In particular, a fluctuating charge-density wave (CDW) has been observed in the cleanest high- $T_{c}$ system $\mathrm{YBa}_{2} \mathrm{Cu}_{3} \mathrm{O}_{6+y} \quad(\mathrm{YBCO})$ for $T^{\star}>T_{\mathrm{CDW}}>T_{c}$ [56-61], but also in $\mathrm{La}_{2-x} \mathrm{Sr}_{x} \mathrm{CuO}_{4}$ [62], which, in principle, may give signatures in the temperature dependence of the Coulomb energy similar to those observed for a superconducting phase transition. Even if Bi2212 and YBCO have structural differences, an analogy may exist, in particular, because charge order is also observed in Bi2212 by STM measurements $[63,64]$.

Such phenomena might very well also occur in the Bi2212 and Bi2223 systems, which are the subject of the present experimental study. However, in principle, it is difficult to tell the difference between fluctuating charge density waves and fluctuating superconducting order on the basis of the temperature trends observed in the optical data. 


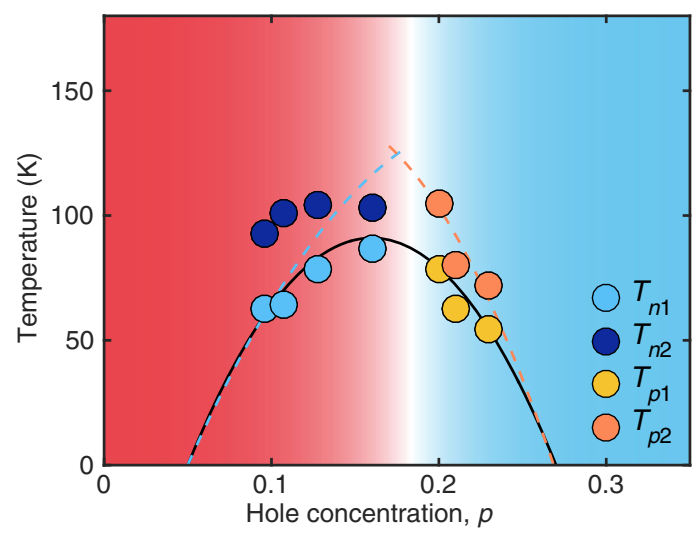

FIG. 6. Phase diagram summarizing the temperature characterizing the step in $\gamma_{C}^{\text {iso }}(T)$ : The temperature of the extremum, coinciding with the superconducting phase transition $\left(T_{n 1} / T_{p 1}\right)$, and the upper limit of the step $\left(T_{n 2} / T_{p 2}\right)$.

Consequently, the weak temperature features that we discuss in the remainder of this section may be attributed to either of these two, as well as other forms of fluctuating order. Figure 6 summarizes all values of $T_{n 1}, T_{n 2}, T_{p 1}$, and $T_{p 2}$ as a function of hole concentration for all $\mathrm{Bi} 2212$ samples and highlights the change of nature of the $T_{n 1}$ extremum in $\gamma_{C}^{\text {iso }}(T)$. It is clear that $T_{n 1}$ and $T_{p 1}$ (light blue and gold circles) can be associated with the critical temperature $T_{c}$ (defined by the empirical Tallon-Presland relation). The transition seen in $\gamma_{C}^{\text {iso }}(T)$ is broadened, especially on the underdoped side, and the resulting curves have a $\Lambda$-like appearance, similar to the Sommerfeld coefficient of underdoped cuprates obtained from specific heat experiments $[37,41-43]$. This aspect is most likely the consequence of fluctuations of the superconducting order above the critical temperature. Concerning $T_{n 2}$ (navy blue circles), the dome shape that it defines, peaked around $p=0.12$, strongly suggests an analogy between this energy scale and the one determined by Nernst effect measurements and associated with a range of temperatures where pair correlations persist above $T_{c}$, but where the phase of the order parameter is strongly fluctuating [48]. Additional indications that in Bi2212, pair correlations persist far above $T_{c}$ come from diamagnetism [47], scanning tunneling spectroscopy [49], specific heat [50], and angle-resolved photo-emission [52,54] experiments. The sign change of the jump of $\gamma_{C}^{\text {iso }}(T)$ when going from underdoped to overdoped samples occurs for $p \approx 0.19$. This doping level coincides with the point where a large body of experimental data indicates the vanishing of the pseudogap [43].

We now turn our attention to the temperature dependence farther above $T_{c}$. The red curves in Figs. 8-15 are phenomenological fits of the function $E_{C}^{\text {iso }}(T)=$ $B+A T^{\alpha}$ to the data above $T_{c}$ (where $B$ and $A$ are some constants). Such a power-law temperature dependence, with $\alpha \sim 2$, is, by and large, described by a constant value

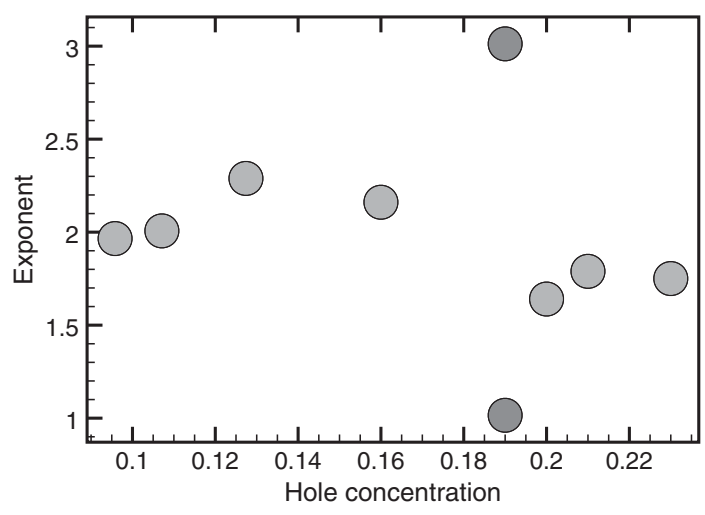

FIG. 7. Exponents of the equation $E_{C}^{\text {iso }}(T)=B+A T^{\alpha}$ obtained from fitting the normal state temperature dependence of the intraisosbestic integrated loss-function intensity, shown in Figs. $8-15$. The two values indicated for the $19 \%$ doped sample (dark gray) refer to different temperature regions.

of $\gamma_{C}^{\text {iso }}(T)$. This behavior finds a natural explanation in the temperature dependence of the free-carrier response at high frequencies, as explained in Ref. [65] (see Appendix H). On a qualitative level, even from visual inspection of these temperature dependences for different dopings, we can already conclude that for the overdoped samples the normal state evolution is less curved than for the underdoped samples. To substantiate this qualitative observation, the evolution from underdoped to overdoped is best illustrated by a plot of the fitted exponents, $\alpha$, as a function of the hole carrier concentration, shown in Fig. 7. The temperature dependence between $T_{c}$ and $300 \mathrm{~K}$ changes progressively as a function of doping. For 19\% doping, the value of the exponent depends strongly on the temperature range fitted, which should be taken as an indication that the temperature dependence is not algebraic for this doping. Interestingly, this is also where the kink at $T_{c}$ changes from positive to negative. The general trend is that the curvature is stronger for underdoped samples, but the exponent returns to the value 2 for the lowest doped material. It should be emphasized that for all dopings, the evolution as a function of temperature above $T_{c}$ is gradual. Nonetheless, comparing the (not too) underdoped and optimally doped samples, the observed behavior may be an indication that upon lowering temperature already far above $T_{c}$, the Coulomb energy of the underdoped samples flows to a lower value than for the overdoped ones. One can then speculate that this is the result of fluctuations of some kind of order parameter (charge density wave, pairing, or other) far above $T_{c}$ in the underdoped cuprates.

On a more detailed level, for samples UD70K, UD83K, OP91K, and possibly Bi2223, we observe in $\gamma_{C}^{\text {iso }}(T)$ (which is negative) a gradual drop for $T>T_{n 1}$, which approaches the constant value for temperatures around $150 \mathrm{~K}$ [66]. This hints at an accelerated saving of Coulomb energy when cooling down below about $150 \mathrm{~K}$ compared to the trend at higher temperatures. 
All in all, the verdict regarding hypothesis $D$ is as follows: We observe possible indications of rather gradual changes of the Coulomb energy in the normal phase, which are possibly associated with the presence of a fluctuating order of some kind in a region above $T_{c}$. First of all, the steps in $\gamma_{C}(T)$ are broadened, especially on the underdoped side, which is almost certainly indicative of a region of fluctuating superconducting order. Second, more subtle and gradual bending of the temperature dependence, especially on the underdoped side, may be due to fluctuations of unknown origin, which disappear gradually as a function of temperature.

\section{SUMMARY AND CONCLUSION}

To summarize, we have measured the evolution as a function of temperature and doping of the loss-function spectra in the infrared-visible spectral range of double- and triple-layer bismuth cuprates. Our experiments indicate that for the overdoped samples, the superconducting phase transition is accompanied by a saving of the Coulomb interaction energy; on the underdoped side, there is an increase of the Coulomb energy below $T_{c}$; and the change of Coulomb energy for $q<0.31 \AA^{-1}$ is about the same size as the condensation energy. This state of affairs calls for studies with other experimental techniques, in particular, electron energy loss spectroscopy, to explore the momentum-dependent structure of these phenomena. Departure of a $T^{2}$ dependence of the measured loss-function data indicates a corresponding temperature dependence of the density-density correlations. Unambiguous assignment to a precursor of superconducting pairing, to another type of correlation, or neither of these two, is not possible at this stage. The S-N difference of the Coulomb energy has similar doping dependence as the total condensation energy. While the latter is in the range of 0 to $2 \mathrm{~K}$ per $\mathrm{CuO}_{2}$ unit, the Coulomb energy varies between -1 and $1 \mathrm{~K}$. Consequently, while it cannot be the whole cause of superconductivity, the Coulomb energy is a major factor in the total energy balance stabilizing the superconducting state. The experiments presented here demonstrate that it is, in principle, possible to determine the subtle changes of Coulomb correlation energy associated with a superconducting phase transition, and they constitute a promising first step in the experimental exploration of the Coulomb correlation energy as a function of momentum and energy.

\section{ACKNOWLEDGMENTS}

The authors thank Mehdi Brandt, Jérémie Teyssier, and Spiros Zanos for technical support. This project was supported by the Swiss National Science Foundation (Project No. 200021-162628). The work at Brookhaven is funded through DOE Contract No. DE-SC00112704. The work at the University of Illinois at Urbana-Champaign was supported as part of the Center for Emergent
Superconductivity, an Energy Frontier Research Center funded by the U.S. Department of Energy, Office of Science, Office of Basic Energy Sciences under Award No. DE-AC02-07CH11358

\section{APPENDIX A: SAMPLE PREPARATION}

Optimally doped single crystals of Bi2223 were grown by the floating zone method. Because of the slow growth kinetics of the 3-layer compound, as compared to the 2-layer one, as well as the need to minimize the formation of Bi2212 intergrowth in the Bi2223 crystals, dedicated growth conditions were chosen. The growth was performed at a very low traveling velocity $(\approx 50 \mu \mathrm{m} / \mathrm{h})$ in a homemade 2-mirror furnace with a steep temperature gradient at the liquid-solid interface $\left(\approx 50^{\circ} \mathrm{C} / \mathrm{mm}\right)$ under a $7 \% \mathrm{O}_{2}-93 \%$ Ar flowing atmosphere. The details of the growth of Bi2223 crystals are reported elsewhere [67]. The resulting as-grown crystals are slightly underdoped. In order to optimally dope and homogenize the oxygen content, cleaved crystals of typical 1-3 mm size were annealed at $500^{\circ} \mathrm{C}$ in 20 bar of $\mathrm{O}_{2}$ for $50 \mathrm{~h}$. As a result, the optimal $T_{c}=110 \mathrm{~K}$ and a transition width as narrow as $\leq 2 \mathrm{~K}$ were obtained. The Bi2223 crystals used for the experiments described in this paper were selected out of dozens as being free of any 2212 traces in the x-ray diffraction pattern and any inflection at about $80 \mathrm{~K}$ in the magnetic susceptibility. This indicates that the amount of Bi2212 intergrowth is well below 1 volume percent. Optimally doped Bi2212 single crystals with $T_{c}=91 \mathrm{~K}$ were grown by using a floating zone method [68]. Underdoped Bi2212 single crystals with a $T_{c}$ of $83 \mathrm{~K}$ were obtained by annealing in a sealed vacuum quartz tube at $450^{\circ} \mathrm{C}$ for three days. Underdoped Bi2212 single crystals with $T_{c}$ 's of $70 \mathrm{~K}$ and $60 \mathrm{~K}$ were obtained by annealing during three days in a vacuum of $10^{-2}$ Torr at $550^{\circ} \mathrm{C}$ and $500{ }^{\circ} \mathrm{C}$, respectively.

\section{APPENDIX B: ELLIPSOMETRY MEASUREMENTS}

In order to accurately determine the temperature and frequency dependence of the dielectric function $\epsilon(\omega, q=0, T)$ in the infrared (from $0.5 \mathrm{eV}$ ), visible, and ultraviolet ranges, we performed spectroscopic ellipsometry using a commercial variable angle spectrometer (Woollam, Inc.). An ultrahigh vacuum cryostat (conflat flanges, no viton) of unique design allows continuous variation of the angle of incidence of the light, $\theta$ between 45 and 90 degrees with the surface normal, without breaking the vacuum. This is achieved by two arms composed of flexible metallic bellows terminated by optical windows that can be set parallel to the light path. Pitch, roll, and yaw of the crystals are controlled with high precision. Sample temperature can be controlled from $10 \mathrm{~K}$ to $400 \mathrm{~K}$. Samples are glued on a conical copper piece allowing rejection of light irrelevant to sample. A cold finger is thermally coupled with copper 
braids to the sample block, which is anchored mechanically to the bottom of the cryostat while remaining thermally isolated from it, ensuring high mechanical stability upon temperature variation. Both a compact turbo-molecular pump and a compact ion pump are mounted directly on top of the cryostat such as to keep pumping resistance to the minimum. After outgassing the cryostat walls by a heatingcooling cycle of several days, the valve to the turbopump is closed, the turbo is switched off, and the ion pump takes over to maintain the base pressure of $10^{-9}$ mbar. These precautions and the ultrahigh vacuum conditions are necessary requirements for stable sample surface conditions during the ellipsometric measurements at low temperatures. Ellipsometry spectra are obtained during cooldown or warm-up in the temperature range from 15 to $300 \mathrm{~K}$ at an average rate of $12 \mathrm{~K}$ per hour. The low noise level needed during the analysis of the present study requires the best possible statistics and imposes the splitting of the spectral range in two: The first covers $0.5-1.5 \mathrm{eV}$, and the second covers $1.5-3.1 \mathrm{eV}$. The angle of incidence was uniformly 70 degrees. All data acquisition and temperature control are computer controlled.

\section{APPENDIX C: PRESENTATION OF THE FULL DATA SET}

In this section, for each of the Bi2212 samples and the Bi2223 sample, we present the loss function and the
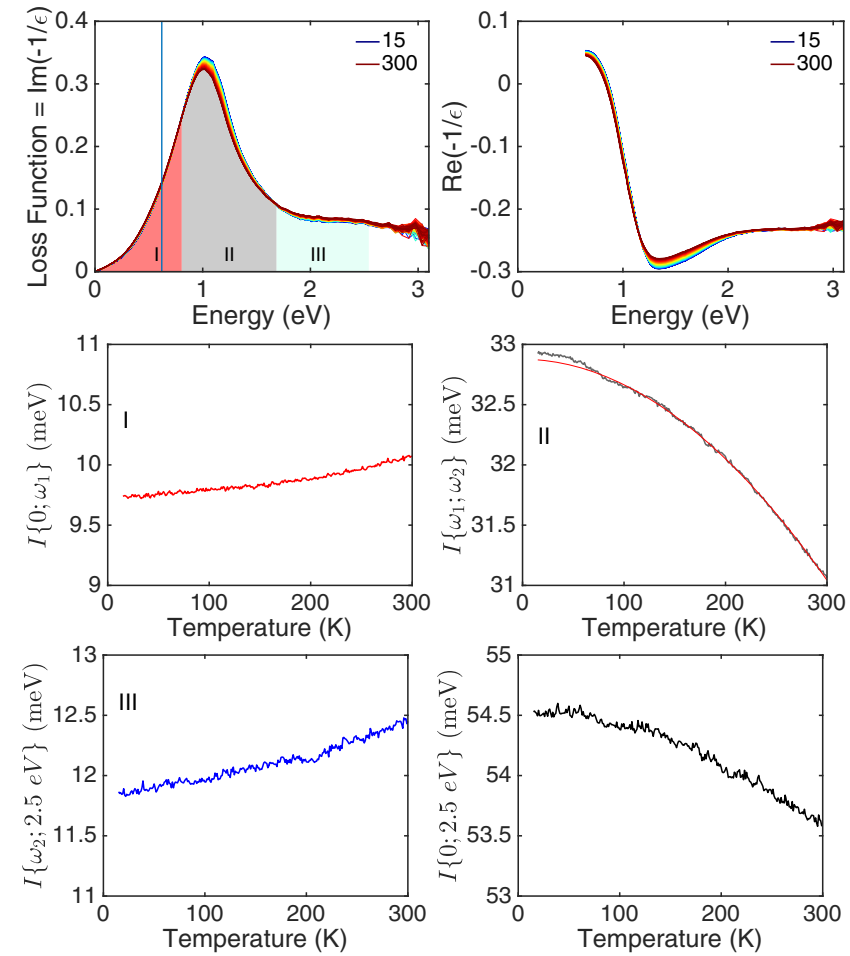

FIG. 8. Loss function and loss-function integrals of sample Bi2212-60-UD. The value of the fitted exponent $\alpha$ is 2.0 (right middle panel). Panel details are further specified in the main text.
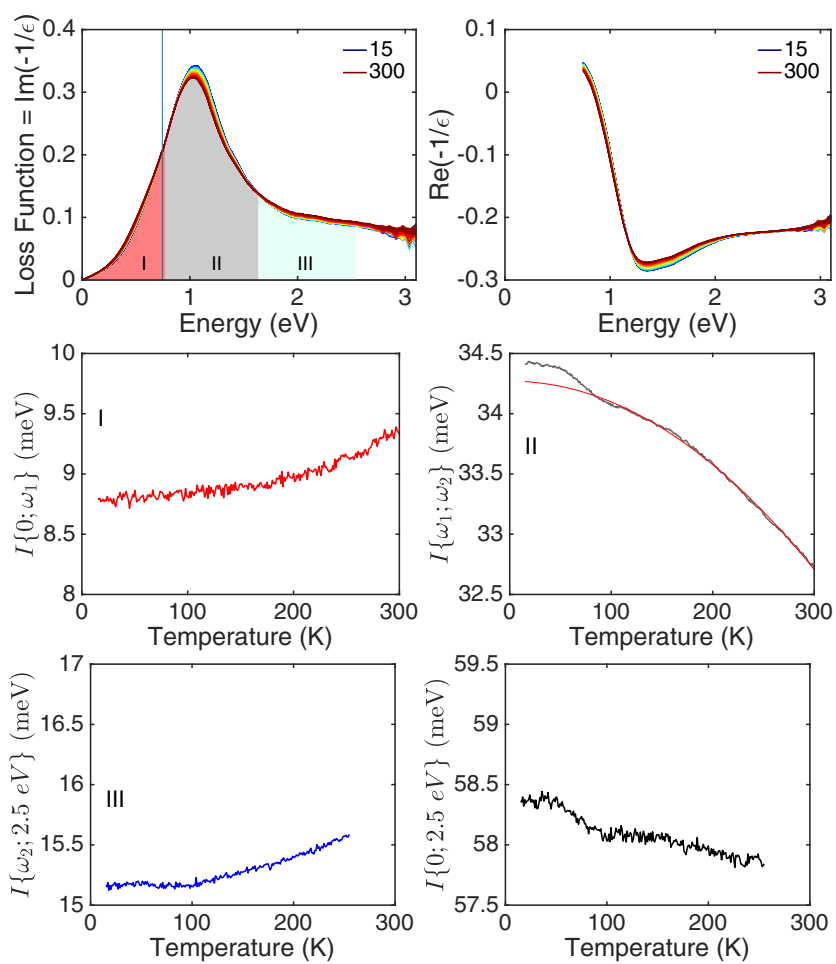

FIG. 9. Sample Bi2212-70-UD. Caption details as in Fig. 8. Because the signal for frequencies in zone 3 was not sufficiently stable above $256 \mathrm{~K}$, the temperature range of the panels on the third line is limited below $256 \mathrm{~K}$. The value of the fitted exponent $\alpha$ is 2.0 .
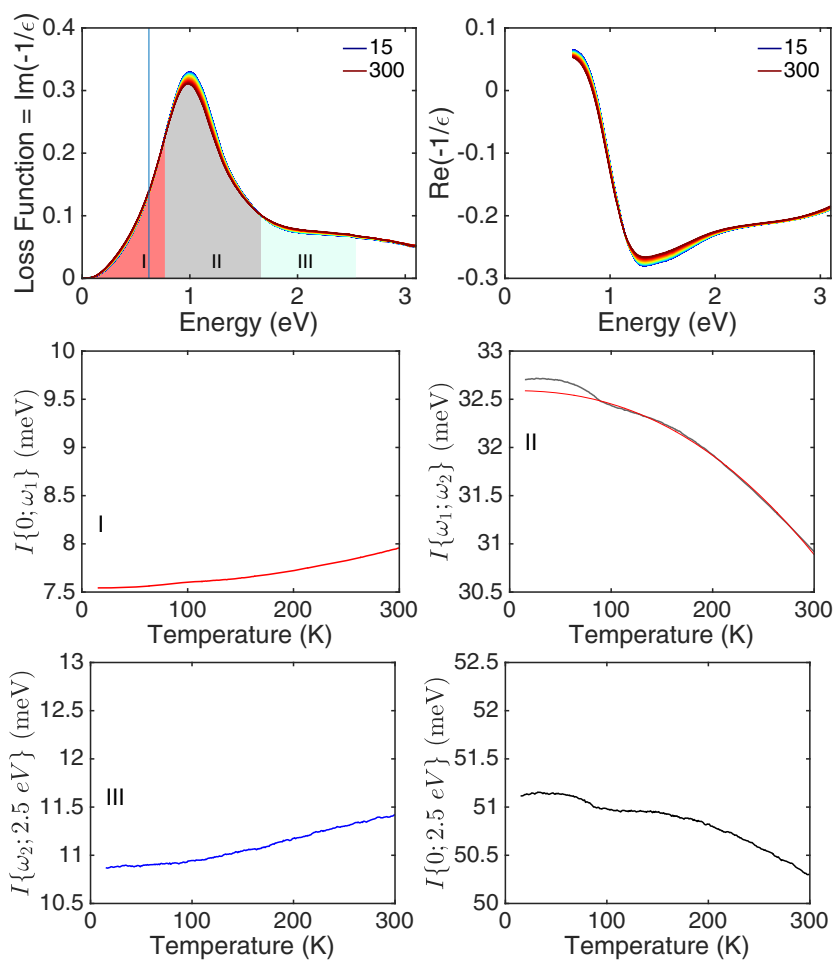

FIG. 10. Sample Bi2212-83-UD. Caption details as in Fig. 8. The value of the fitted exponent $\alpha$ is 2.3. 

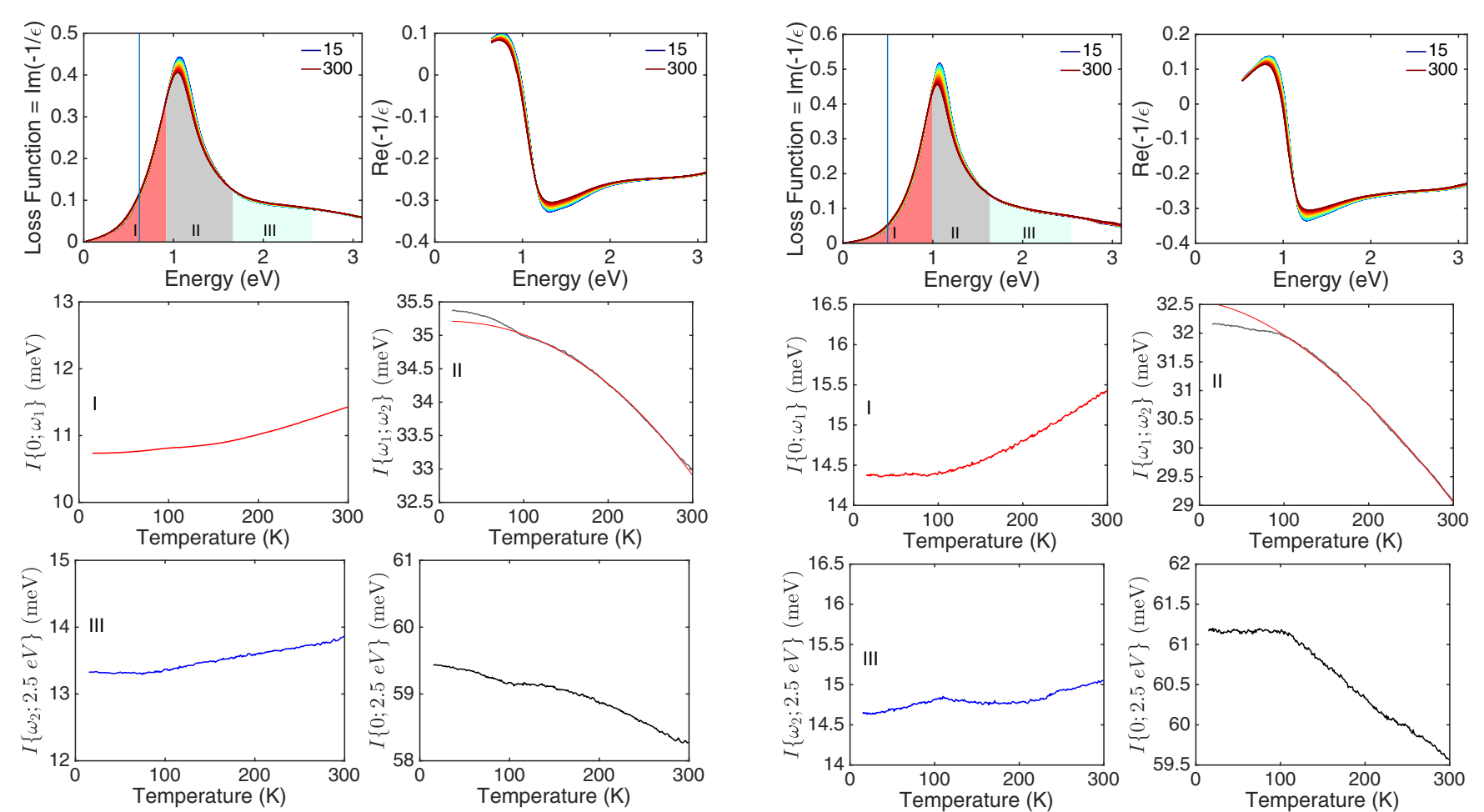

FIG. 11. Sample Bi2212-91-OpD. Caption details as in Fig. 8. The value of the fitted exponent $\alpha$ is 2.2.
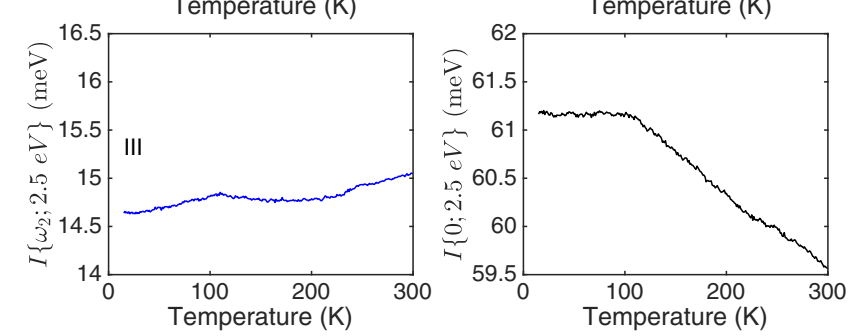

FIG. 13. Sample Bi2212-81-OD. Caption details as in Fig. 8. The value of the fitted exponent $\alpha$ is 1.6.
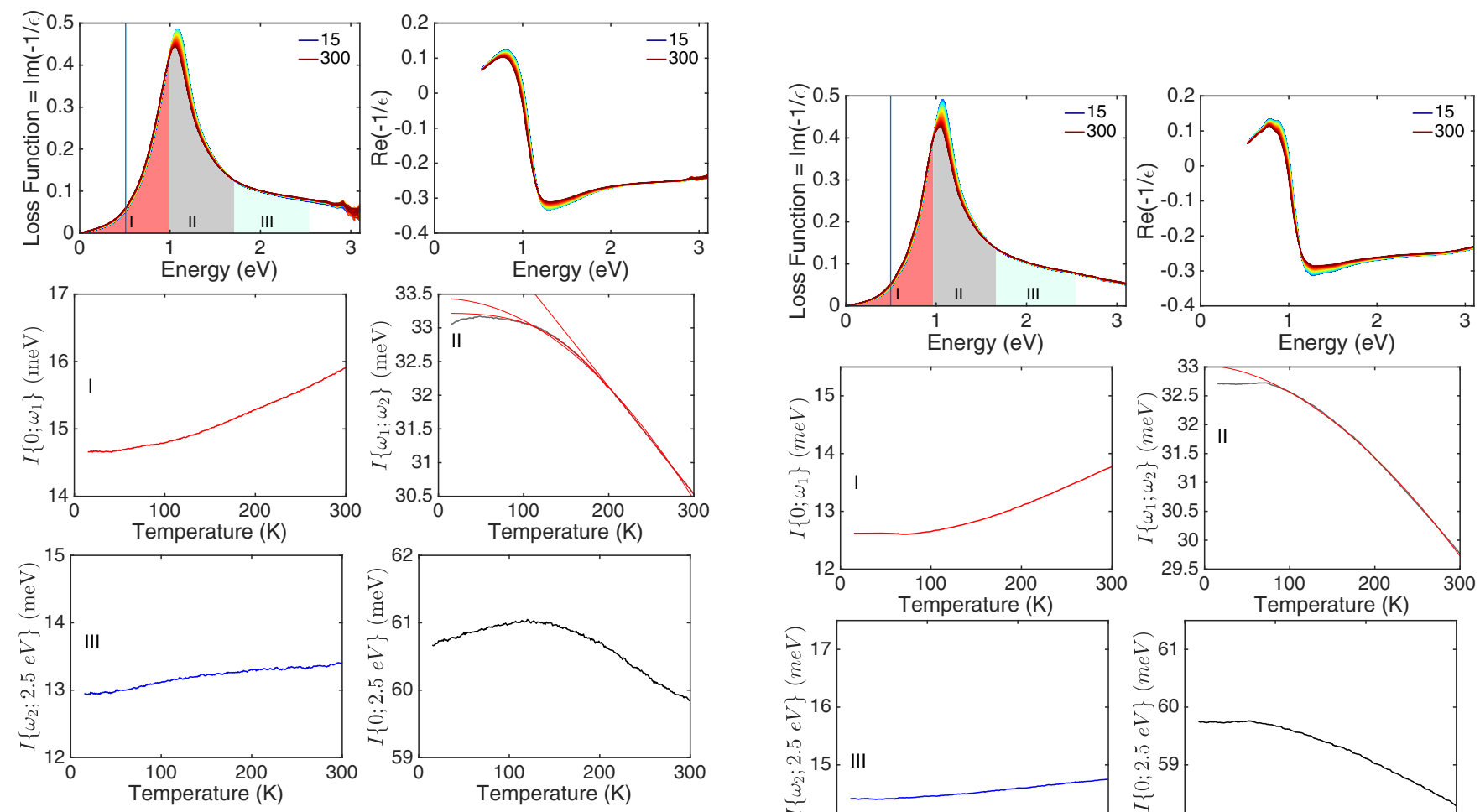

FIG. 12. Sample Bi2212-84-OD. Caption details as in Fig. 8. The value of the fitted exponent $\alpha$ is 2 when fitting down to $T_{c}$ and 1 and 3 when splitting into $T_{c}-200 \mathrm{~K}$ and $200-300 \mathrm{~K}$ windows.
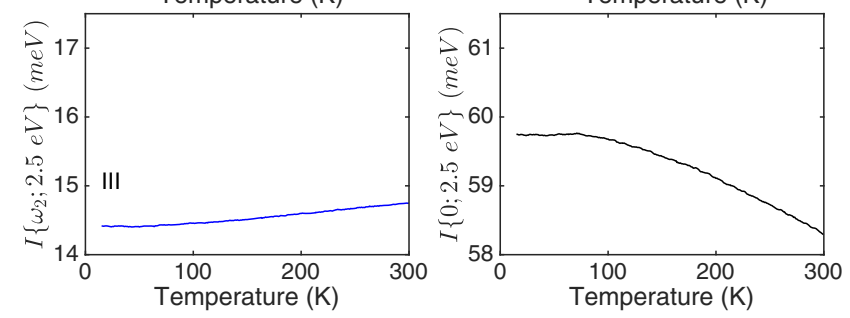

FIG. 14. Sample Bi2212-70-OD. Caption details as in Fig. 8. The value of the fitted exponent $\alpha$ is 1.8 . 

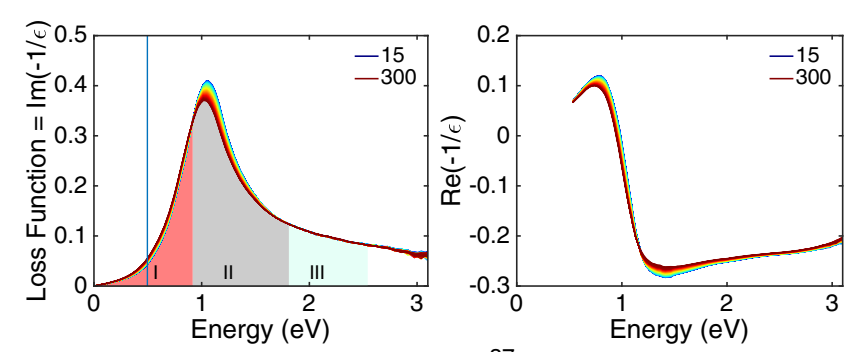

perpendicular to the sample surface is $\epsilon_{c}$, and along the sample surface, we have $\epsilon_{a}$. In this case, the Fresnel equations give

$$
\eta=\frac{\left(1+\delta-\epsilon_{a}\right) \cos \theta \sqrt{\epsilon_{a}-\sin ^{2} \theta}}{\epsilon_{a} \cos ^{2} \theta-(1+\delta)\left(\epsilon_{a}-\sin ^{2} \theta\right)},
$$

where

$$
\delta=\sqrt{\frac{1-\epsilon_{a}^{-1} \sin ^{2} \theta}{1-\epsilon_{c}^{-1} \sin ^{2} \theta}}-1 .
$$

The $\epsilon_{a}(\omega)$ spectra can be obtained from the combination of $\rho(\omega)$ and $\epsilon_{c}(\omega)$ using the method described below. In the isotropic case $\left(\epsilon_{a}=\epsilon_{c} \equiv \epsilon\right)$, we have $\delta=0$, and Eq. (D2) simplifies to

$$
\eta=\frac{\cos \theta \sqrt{\epsilon-\sin ^{2} \theta}}{\sin ^{2} \theta}
$$

Given the value of $\eta$ measured by ellipsometry, we can then calculate the dielectric function $\epsilon$ using the relation

$$
\epsilon=\sin ^{2} \theta+\frac{\eta^{2} \sin ^{4} \theta}{\cos ^{2} \theta} .
$$

loss-function integrals as a function of temperature in different frequency domains. In the top panels of Figs. $8-15$ are displayed: imaginary (left panel) and real (right panel) parts of $-\epsilon(\omega)^{-1}$ for selected temperatures. The curves in the interval on the left of the vertical blue line are Drude-Lorentz oscillator fits to the experimental complex loss function in the range $0.6-3.1 \mathrm{eV}$. The second and third rows from left to right are as follows: Integral of the loss function of the red, gray, blue, and total areas as a function of temperature. The right middle panel shows power-law temperature dependence $B+A T^{\alpha}$, fitted to the data in the normal state.

\section{APPENDIX D: $c$-AXIS CORRECTION}

Ellipsometry determines the ratio of $p$-polarized over $s$-polarized reflectivity coefficients, $\rho=r_{p} / r_{s}$, which in turn is a function of the tensor elements of the dielectric function and the angle of incidence relative to the surface normal, $\theta$. This relation is given by the Fresnel equations. In the interest of compactness of notation, we define

$$
\eta \equiv \frac{\rho-1}{\rho+1}
$$

which, from now on, we treat as the primary experimental ellipsometric quantity. Here, we consider the ellipsometric data for an optical uniaxial material with the optical axis perpendicular to the sample surface. The dielectric constant

We begin by noting that, according to Aspnes [69], even if the material is optically anisotropic, Eq. (D5) provides a good approximation of the tensor element of the dielectric constant along the intersection of the plane of reflection and the sample surface. For an uniaxial material with the optical axis perpendicular to the sample surface Eq. (D2) can be solved iteratively, starting from Aspnes' zeroth order solution, Eq. (D5), i.e., $\epsilon_{a, 0}=\epsilon$. The speed of convergence is controlled by the smallness of $\delta_{j}$ defined in Eq. (D3) (with $\epsilon_{a, j}^{-1}$ instead of $\epsilon_{a}^{-1}$ ). The full solution of Eq. (D2) is obtained by substituting $\epsilon_{a, 0}$ in the right-hand side of the expression,

$$
\epsilon_{a, j+1}=\sin ^{2} \theta+\frac{\eta^{2}}{\cos ^{2} \theta}\left[\sin ^{2} \theta-\frac{\delta_{j} \epsilon_{a, j}}{1-\epsilon_{a, j}+\delta_{j}}\right]^{2} .
$$

The process continues by resubstituting $\epsilon_{a, j+1}$ in the righthand side of the expression, which is reiterated until convergence is reached. Convergence typically takes less than 20 cycles, as illustrated by the example shown in Fig. 17. The $c$-axis optical constants have been reported in Ref. [70] (Bi2212) and Refs. [33,71] (Bi2223) and have been found to be essentially independent of temperature for frequencies above $0.5 \mathrm{eV}$. Furthermore, $\operatorname{Re} \epsilon_{c}(\omega) \sim 3-5$, and $\operatorname{Im} \epsilon_{c}(\omega)$ is very small. Because of crystal imperfections, some $a$-axis admixture may have occurred in aforementioned experiments, during which the measured $\epsilon_{c}(\omega)$ may have been underestimated. Moreover, quite generally, 

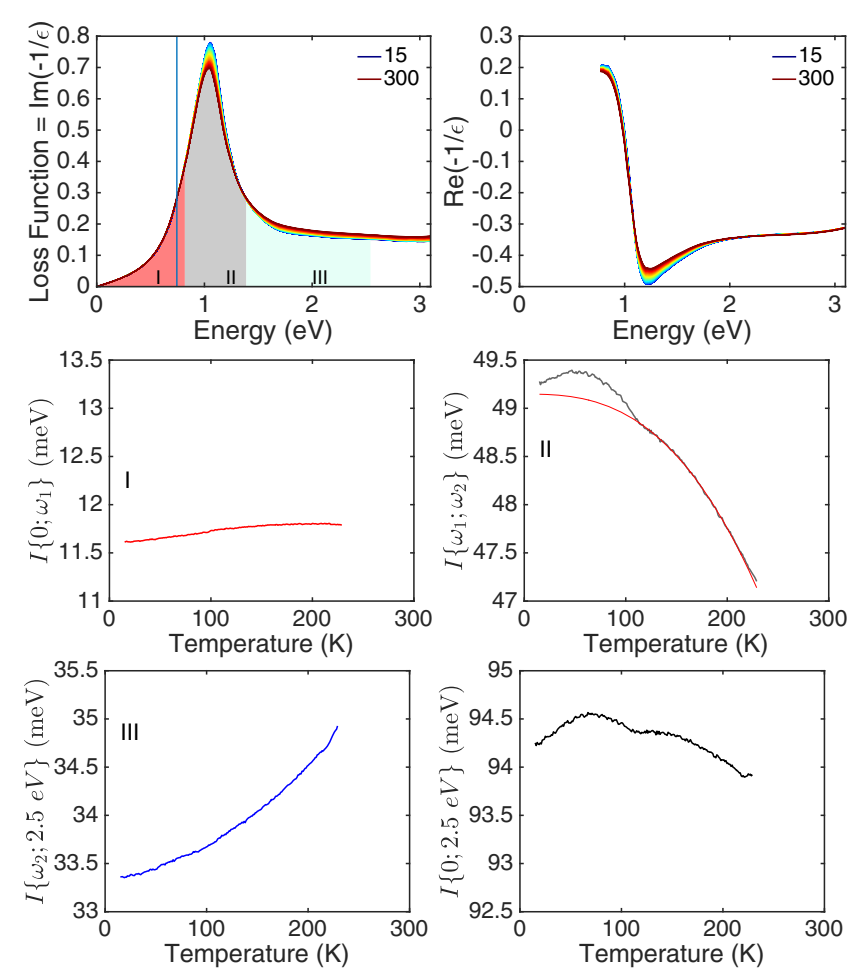

FIG. 16. Sample Bi2223-110-OP. Caption details as in Fig. 8. The value of the fitted exponent $\alpha$ is 2.7. The evolution as a function of temperature above $220 \mathrm{~K}$ was not fully reproducible because of a combination of factors having to do with small crystal size and instrument drift. The temperature range for this sample is therefore limited below $220 \mathrm{~K}$.

the bound-charge polarizability in the cuprates as obtained from $a b$-plane experiments corresponds to $\epsilon_{b}=4.5 \pm 0.5$ for Bi2212 and Bi2223. The anisotropy of the bound charge polarizability is known to be small in the cuprates, so $\epsilon_{c}(\omega)$ (for which the free-carrier contribution is negligible) should be near 4.5. Anomalous spectral weight changes below $T_{c}$ in the $c$-axis response of underdoped cuprates has been discussed, reported, and analyzed in Refs. [31,72-76]. One may then wonder whether a $T$ dependence of the $c$-axis response, even a weak one, may interfere with the relatively small changes of the in-plane loss-function spectra that are reported here. To investigate this possible influence, we analyze the case of optimally doped Bi2223. The same pseudo-dielectric function is fed into the procedure described above, where this time $\epsilon_{c}(\omega, T)$ used in Eq. (D6) is measured by ellipsometry [77]. The resulting loss function is shown in Fig. 18(a). Apart from a general level increase, there are no qualitative distinctions between this loss function and the one that we obtained with $\epsilon_{c}=3.5$ shown in Fig. 16. The temperaturedependent loss-function integrals in Fig. 18(b) yield extremely close temperature dependence and so do the corresponding Sommerfeld coefficients in Fig. 18(c) with matching characteristic temperatures. This has motivated us to adopt, for the $c$-axis correction described above,
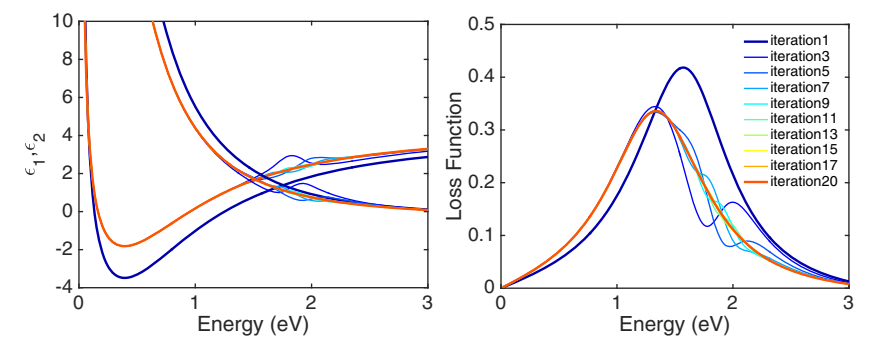

FIG. 17. Example of the $c$-axis correction obtained in 20 iterations: dielectric function (left panel) and corresponding loss function (right panel).
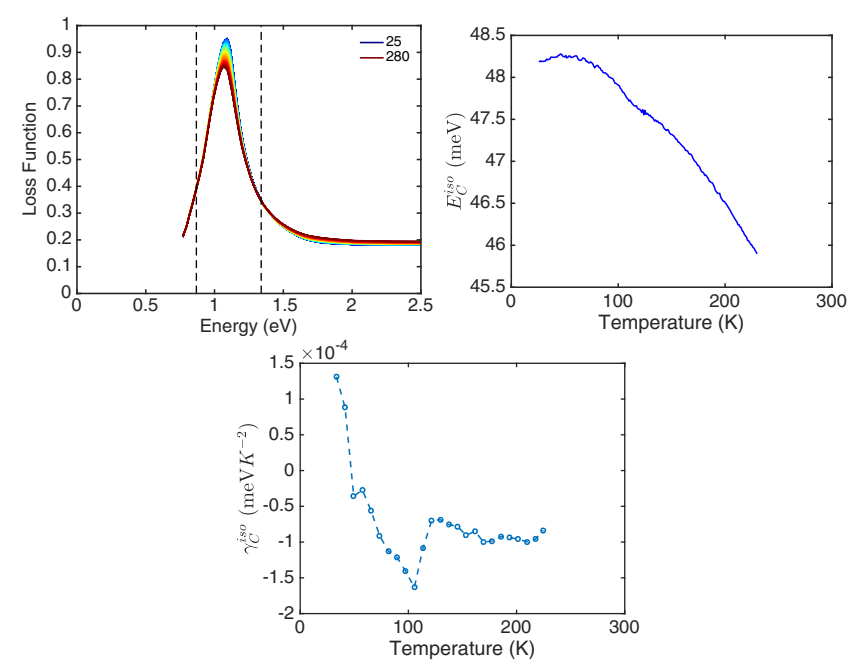

FIG. 18. Bi2223 $c$-axis correction using $\epsilon_{c}(\omega, T)$ from Ref. [77].

$\epsilon_{c}=4.5$ for Bi2212 and $\epsilon_{c}=3.5$ for Bi2223 throughout the frequency and temperature ranges from 0.5 to $3.1 \mathrm{eV}$.

\section{APPENDIX E: LOW-ENERGY DRUDE-LORENTZ EXTRAPOLATION}

It was mentioned in Sec. III that for a list of reasons, our analysis uses a spectral range limited by two isosbestics points, $\omega_{1}$ and $\omega_{2}$, displayed in Fig. 19 for the under-doped Bi2212-83K sample. We show here that, in fact, the $0-\hbar \omega_{1} \mathrm{eV}$ part of the loss function amounts to a marginal contribution to the partial Coulomb energy. To remain as
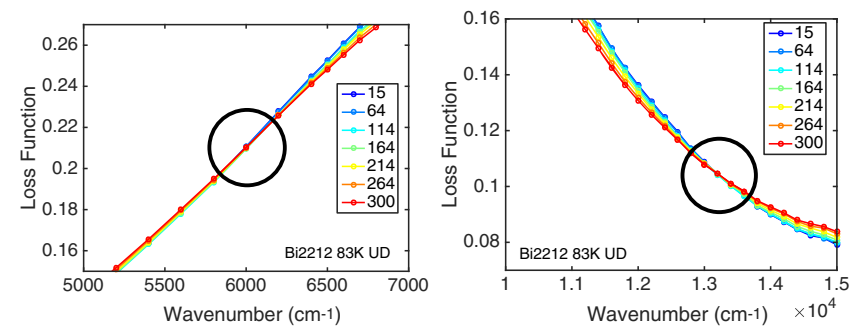

FIG. 19. Isosbestics points for Bi2212 83K UD. 

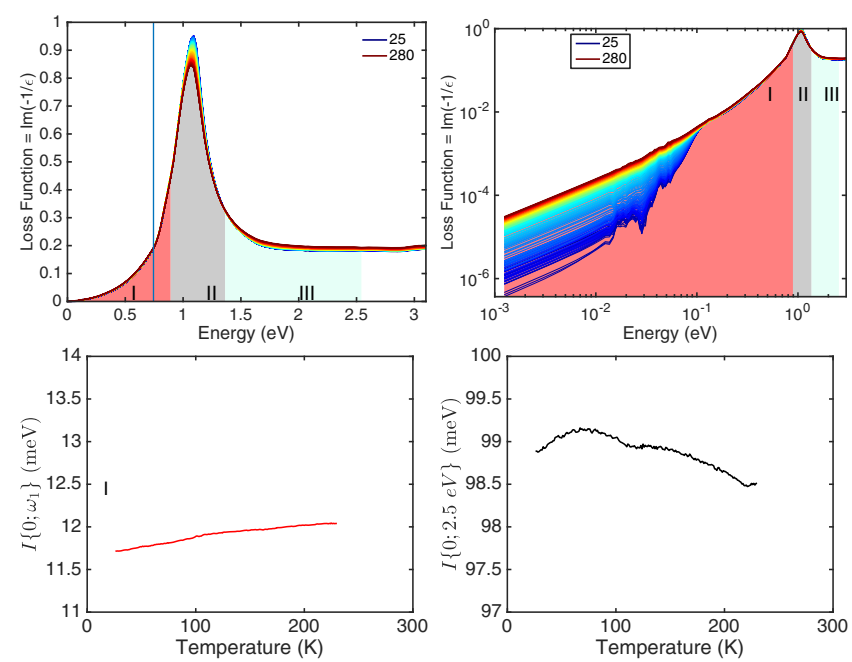

FIG. 20. Bi2223 optimally doped with an extended low-energy region from Ref. [77].

close as possible to experimental input, we consider the Bi2223 loss function obtained with a $c$-axis data correction to which the low-energy part of the spectrum is complemented by far-infrared data [77] as shown in Fig. 20(a). This region, situated below our measurement range, contains all previously reported superconductivity induced changes of temperature-dependent optical properties at photon energies, above the superconducting gap. However, these do not appear as strong features in the loss function at all: These spectral details are only distinguishable from a Drude-Lorentz fit (shown in Fig. 21) when displaying the data in a log-log scale, as shown in Fig. 20(b). The temperature dependence of the loss-function integral over the $0-\hbar \omega_{1}$ and the $0-2.5 \mathrm{eV}$ regions is shown in the second row of Fig. 20. It is obvious, when comparing the $0-2.5 \mathrm{eV}$ integral with the one carried out on
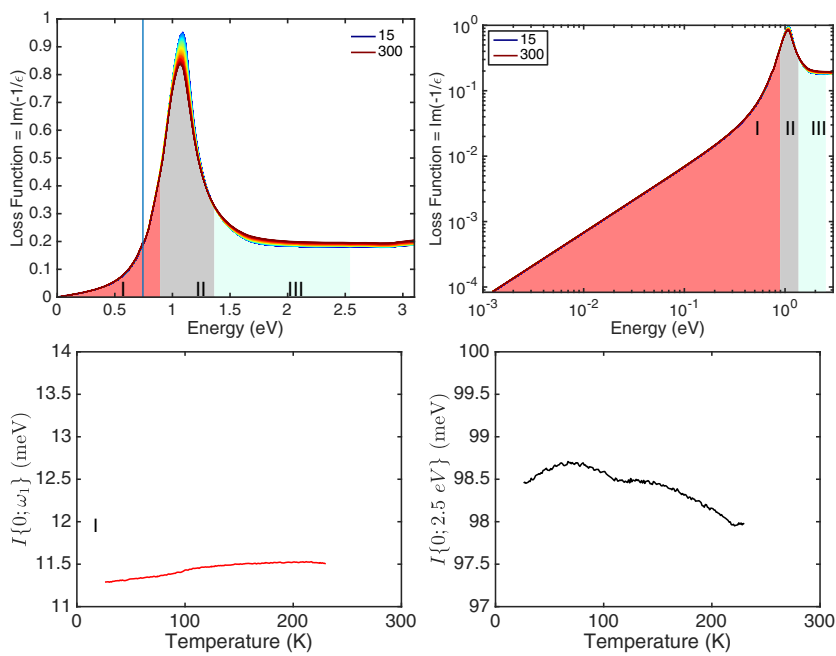

FIG. 21. Bi2223 optimally doped with the low-energy region extrapolated. extrapolated data shown in Fig. 21, that aside from an $\sim 0.5 \mathrm{meV}$ offset, the temperature dependence extracted from the two methods is identical. Additionally, one can also notice that the temperature dependence of the data at low energy is actually irrelevant to the general behavior of the $0-2.5 \mathrm{eV}$ integral of the loss function.

\section{APPENDIX F: SCREENING OF THE COULOMB INTERACTION IN A LAYERED MATERIAL}

In this paper, we use the data obtained in optical experiments, plus the "extrapolation assumption," to infer something about the changes in the expectation value of the long-wavelength (but not very long) part of the interconduction electron Coulomb interaction $E_{C}$. While in an isotropic 3D material this procedure is straightforward (see below), the strongly layered nature of the cuprates gives rise to a complication, as follows: As we will verify below, in a layered material with inter(multi)layer separation $s$, the screening of the Fourier component $V_{q}$ of the Coulomb interaction depends qualitatively on $q$ : For $q s \ll 1$, it is of the standard "bulk 3D" form and is completely taken into account by a $q$-independent dielectric constant $\epsilon(\omega)$; for $q s \gtrsim 1$ the effect is more complicated; and for $q s \gg 1$, it is represented by a $q$-dependent "pseudo-dielectric constant" $\epsilon_{p s}=1+[q s / 2][\epsilon(\omega)-1]$. This is because, while in 3D the Fourier component is proportional to $q^{-2}$, in $2 \mathrm{D}$ it is $\propto q^{-1}$. Since in the MIR scenario the main contribution to $E_{C}$ comes from $q s \gtrsim 1$ with a strictly two-dimensional $q$ (but the optics measures the dielectric tensor for $q s \ll 1$ where $q$ is three dimensional), some care is necessary.

In the following, we assume until further notice, as in Refs. [3,4], (a) that any screening of the inter-Coulomb energy by the ionic cores is itself three dimensional and hence may be represented by a $q$ - and $\omega$-independent constant $\epsilon_{\mathrm{sc}}$, and (b) that intermultilayer (inter-unit-cell) tunneling is negligible and hence that, in the frequency range of interest, the experimentally observed $(q=0)$ $c$-axis dielectric function is some $\omega$-independent constant. The effect of relaxing these assumptions will be briefly discussed in Appendix G. For pedagogical simplicity, we give the explicit discussion for a single-plane cuprate; the generalization to the actual (bilayer) case of interest is straightforward and will be indicated where necessary.

The general statement following from the fluctuationdissipation theorem is that at $T=0$ (which we adopt throughout this subsection), the full susceptibility $\chi(q, \omega)$ and the qth Fourier component of the Coulomb interaction are related as

$$
E_{C}^{\mathbf{q}}=\frac{\hbar}{2 \pi} \operatorname{Im} \int_{0}^{\infty} V_{\mathbf{q}} \chi(\mathbf{q}, \omega) d \omega
$$

where $V_{\mathbf{q}}$ is the Fourier transform of the bare Coulomb interaction. For a bulk 3D sample, this implies a direct relation to the longitudinal dielectric function 


$$
E_{C}^{\mathbf{q}}=\frac{\hbar}{2 \pi} \operatorname{Im} \int_{0}^{\infty} d \omega \frac{-1}{\epsilon(\mathbf{q}, \omega)}
$$

However, $\epsilon(\mathbf{q}, \omega)$ characterizes the dielectric response of a 3D material; consequently, Eq. (F2) cannot be directly applied to, e.g., the case of a two-dimensional conducting plane. The general formula irrespective of the geometry is given by

$$
E_{C}^{\mathbf{q}}=\frac{\hbar}{2 \pi} \operatorname{Im} \int_{0}^{\infty} d \omega \frac{-1}{1+V_{\mathbf{q}} \chi^{(0)}(\mathbf{q}, \omega)},
$$

where $\chi^{(0)}(\mathbf{q}, \omega)$ is defined by the relation

$$
\chi(\mathbf{q}, \omega)^{-1}=\chi^{(0)}(\mathbf{q}, \omega)^{-1}+V_{\mathbf{q}} .
$$

Note that this is a definition of $\chi^{(0)}(\mathbf{q}, \omega)$, which, despite the resemblance to the random phase approximation, does not rely on the validity of that approximation. However, we assume, for present purposes, that in the $(\mathbf{q}, \omega)$ region of interest $\left(q \neq 0, q_{z}=0\right.$, where $q$ and $q_{z}$ are the $a b$-plane and $c$-axis components, respectively) $V \chi^{(0)}$ is a function only of $\omega$ (this subsumes the "extrapolation assumption"), and that for the $c$-axis component $\left(q_{z} \neq 0, q=0\right)$ $V \chi^{(0)}=0$, and thus the $c$-axis component does not contribute to Eq. (F3); we return to it in Appendix G.

The three-dimensional Fourier transform of the Coulomb potential in a layered electron gas is $[78,79]$

$$
V_{\mathbf{q}}=\frac{e^{2} s}{2 \epsilon_{0} q} \frac{\sinh q s}{\cosh q s-\cos q_{z} s} .
$$

In the limit $|\mathbf{q}| s \ll 1$, this is just $e^{2} /\left(\epsilon_{0}|\mathbf{q}|^{2}\right)$, and hence $E_{C}^{\mathbf{q}}$ is simply the integral over the familiar loss function $\operatorname{Im} \epsilon(\mathbf{q}, \omega)^{-1}$. However, the regime that dominantly contributes to the overall Coulomb energy is $q s \gtrsim 1$. If we make the approximation $q s \gg 1$, then for any $q_{z}, V_{q}$ is approximately given by $e^{2} s /\left(2 \epsilon_{0} q\right)$, so after summation over $q_{z}$, we find the "per-plane" result

$$
E_{C}^{\mathbf{q}}=\frac{\hbar}{2 \pi} \operatorname{Im} \int_{0}^{\infty} d \omega \frac{-1}{1+(q s / 2)\left[\epsilon_{a}(\omega)-1\right]} .
$$

In Appendix G, a more general formula [Eq. (G9)] for $E_{C}^{\mathbf{q}}$ is given, and it is shown that after summation over $q_{z}$, the result integrated over $q$ up to a cutoff $q_{0}$ coincides with Eq. (F6) provided $q_{0} s \gg 1$.

However, we wish to calculate the value of the screened Coulomb interaction between the conduction electrons. This can be done simply by replacing $V_{q}$ by $V_{q}^{\mathrm{sc}}=V_{q} / \epsilon_{\mathrm{sc}}$, where $\epsilon_{\mathrm{sc}}$ is the frequency- and wave-vector-independent dielectric constant due to screening of the conduction electrons by the ionic cores (recall we are assuming this screening to be three dimensional, i.e., uniformly due to the whole unit cell). In the limit $q s \ll 1$, the effect is simply to multiply the expression (F3) by an overall factor of $\epsilon_{\mathrm{sc}}$. We may see this more explicitly as follows: The general form of the Hamiltonian of a metal is [see Eq. (2)]

$$
\hat{H}=\hat{T}+\hat{U}+\hat{V}_{C}
$$

where $\hat{T}$ is the kinetic energy, $\hat{U}$ the crystal potential, and

$$
\hat{V}_{C}=\frac{1}{2} \sum_{\mathbf{q}} V_{\mathbf{q}} \hat{\rho}_{\mathbf{q}} \hat{\rho}_{-\mathbf{q}} .
$$

The dielectric function of such a many-electron system is

$$
\epsilon(\mathbf{q}, \omega)=1+V_{\mathbf{q}} \chi^{(0)}(\mathbf{q}, \omega) .
$$

Often one is interested in the free-carrier properties, in which case it is useful to integrate out the degrees of freedom having to do with the "bound charge," i.e., the interband transitions. This distinction is meaningful when the interband transitions are well separated from the intraband degrees of freedom, which is actually the case in the cuprates. The dielectric constant can be split as follows:

$$
\epsilon(\mathbf{q}, \omega)=1+S(\mathbf{q}, \omega)+V_{\mathbf{q}} \chi^{(0)}(\mathbf{q}, \omega),
$$

where $S(\mathbf{q}, \omega)$ subsumes all bound charge terms. At low frequencies and small $q$, we have

$$
1+S(\mathbf{q}, \omega)=\epsilon_{\mathrm{sc}} .
$$

We will neglect $q$ and $\omega$ dependencies up to frequencies $\omega_{b}$, which is some high-energy-scale representative of the interband transitions. The dielectric function at low frequencies becomes

$$
\epsilon(\mathbf{q}, \omega)=\epsilon_{\mathrm{sc}}\left\{1+V_{\mathbf{q}}^{\mathrm{sc}} \chi^{(0)}(\mathbf{q}, \omega)\right\},
$$

where $V_{\mathbf{q}}^{\text {sc }}=V_{\mathbf{q}} / \epsilon_{\mathrm{sc}}$. The effective low-energy Hamiltonian is

$$
\hat{H}^{\mathrm{eff}}=\hat{K}+\hat{V}_{C}^{\mathrm{sc}}
$$

where the first term describes the free charge carrier band dispersion and the second one the bare Coulomb interaction screened by the aforementioned bound charges, so

$$
\hat{V}_{C}^{\mathrm{sc}}=\frac{1}{2} \sum_{\mathbf{q}} V_{\mathbf{q}}^{\mathrm{sc}} \hat{\rho}_{\mathbf{q}}^{f} \hat{\rho}_{-\mathbf{q}}^{f}
$$

Consequently, the relation between low-energy susceptibility taking into account the screened interaction $\chi^{\mathrm{sc}}(\mathbf{q}, \omega)$ and $\chi^{(0)}(\mathbf{q}, \omega)$ becomes 


$$
\chi^{\mathrm{sc}}(\mathbf{q}, \omega)^{-1}=\chi^{(0)}(\mathbf{q}, \omega)^{-1}+V_{\mathbf{q}}^{\mathrm{sc}}
$$

The fluctuation dissipation theorem [24] provides the relation between the screened Coulomb interaction and the limited range susceptibility integral

$$
\frac{\hbar}{\pi} \int_{0}^{\omega_{b}} \operatorname{Im} \chi^{\mathrm{sc}}(\mathbf{q}, \omega) d \omega=\left\langle\hat{\rho}_{\mathbf{q}}^{f} \hat{\rho}_{-\mathbf{q}}^{f}\right\rangle
$$

from which, with the help of Eqs. (F12) and (F16),

$$
E_{C}^{\mathrm{mir}}=\left\langle\hat{V}_{C}^{\mathrm{sc}}\right\rangle=\frac{\hbar \epsilon_{\mathrm{sc}}}{2 \pi} \sum_{\mathbf{q}} \int_{0}^{\omega_{b}} \operatorname{Im} \frac{-1}{\epsilon(\mathbf{q}, \omega)} d \omega .
$$

Equation (F17) tells us that the loss-function integral, when carried out over the free-carrier part of the response, probes a fraction $1 / \epsilon_{\mathrm{sc}}$ of the Coulomb interaction between the free charge carriers, which-compared to the bare Coulomb interaction - is already reduced by an additional factor $1 / \epsilon_{\mathrm{sc}}$. To verify that no double counting of $1 / \epsilon_{\mathrm{sc}}$ has occurred, we reformulate the individual $q$-terms of the lhs and rhs of Eq. (F17),

$\frac{\hbar}{2 \pi} \int_{0}^{\omega_{b}} \frac{V_{\mathbf{q}}^{\mathrm{sc}} \operatorname{Im} \chi^{(0)}(\mathbf{q}, \omega)}{\left|1+V_{\mathbf{q}}^{\mathrm{sc}} \chi^{(0)}(\mathbf{q}, \omega)\right|^{2}} d \omega=\frac{1}{2}\left\langle V_{\mathbf{q}}^{\mathrm{sc}} \hat{\rho}_{\mathbf{q}}^{f} \hat{\rho}_{-\mathbf{q}}^{f}\right\rangle$,

which in the weak coupling limit $\left(V_{\mathbf{q}} \rightarrow 0\right.$, so that the denominator $\rightarrow 1$ ), returns the fluctuation-dissipation theorem for the noninteracting system

$$
\frac{\hbar}{\pi} \int_{0}^{\omega_{b}} \operatorname{Im} \chi^{(0)}(\mathbf{q}, \omega) d \omega=\left\langle\hat{\rho}_{\mathbf{q}}^{f} \hat{\rho}_{-\mathbf{q}}^{f}\right\rangle
$$

as expected. The remaining fraction $\left(1-1 / \epsilon_{\mathrm{sc}}\right)$ of the screened interaction energy is recovered in the loss function in the range of interband transitions. Since the interband region is typically smeared out over several tenths of $\mathrm{eV}$, the corresponding signatures are small and very difficult to detect experimentally.

In the more relevant case of a layered system with $q s \gg 1$, we can go through the same argument, but we must now bear in mind that $V_{q}^{\mathrm{sc}} \chi(q, \omega)$ is no longer equal to $\left[\epsilon(\omega) / \epsilon_{\mathrm{sc}}-1\right]$ but rather to $\left[q s \epsilon(\omega) / \epsilon_{\mathrm{sc}}-1\right]$. Thus, we recover Eqs. (4.1.4) and (4.1.5) of Ref. [4], and hence Eq. (14) of the main text. Finally, if we relax the assumption that the core screening is three dimensional (for example, assume that it comes only from the highly planar array of intralayer Cu's and O's), the effect is simply to replace the quantity $\epsilon_{\mathrm{sc}}$ in Eq. (14) by the relevant $\epsilon_{\mathrm{sc}}(q)$, which may have a substantial $q$ dependence but, in practice, does not make much difference for the computed value of $E_{C}^{\mathrm{mir}}$.

\section{APPENDIX G: $c$-AXIS CONTRIBUTION IN A LAYERED MATERIAL}

For the $c$-axis, the free-carrier spectral weight is very low. The transition from normal to superconducting state is characterized by the appearance of a Josephson plasmon. For the Bi2212 materials, the Josephson plasma frequency is much lower than the (already low) frequency observed in single-layer T12201 [73]; in fact, the exact value is not known since it is below the experimental window of infrared spectroscopy. However, Zelezny et al. [76] observed a temperature dependence in the optical phonon range, which they attributed to the transverse optical Josephson plasmon [75]. In order to have an influence on the ellipsometric data in the range of the $a b$-plane plasmon, it would be necessary that the optical spectral weight associated with such a plasmon is transferred from high energy. There are no indications for this; in fact, the absence of reflectivity changes in the near infrared in these compounds (as discussed, for example, in Appendix D) rather suggests that this spectral weight is reshuffled within the infrared range. If we nonetheless model the $c$-axis dielectric function with a $400 \mathrm{~cm}^{-1}$ plasma frequency which disappears in the normal phase (equivalent to a transfer of the associated optical spectral weight to infinite frequency), we obtain a strongly overestimated upper bound of the $c$-axis contribution to the Coulomb energy. To simulate the effect on the optical properties, we use the following expressions:

$$
\begin{aligned}
\epsilon_{c}(\omega, T) & =\epsilon_{\mathrm{sc}}-\frac{\omega_{p c}^{2} n_{s}(T)}{\omega^{2}}, \\
n_{s}(T) & = \begin{cases}1-\left(T / T_{c}\right)^{2} & T \leq T_{c} \\
0 & T>T_{c},\end{cases}
\end{aligned}
$$

with the parameters $\omega_{p c} / 2 \pi c=400 \mathrm{~cm}^{-1}$ and $\epsilon_{\mathrm{sc}}=4.5$. We furthermore assume, as per the MIR scenario, that the value of the loss function can be extrapolated up to an upper limit $q_{0}$, and for larger $q$, it has a negligible contribution to the $\mathrm{S}-\mathrm{N}$ difference of the Coulomb energy. This yields the $q$-averaged effective Coulomb energy in the MIR scenario [see Eqs. (F17) and (F2)],

$$
E_{C}^{\mathrm{mir}}=\frac{\hbar \epsilon_{\mathrm{sc}} a^{2} s}{8 \pi^{3}} \operatorname{Im} \int_{0}^{\omega_{b}} d \omega \int_{0}^{q_{0}} q d q \int_{-\pi}^{\pi} d q_{z} \frac{-1}{\epsilon(\mathbf{q}, \omega)},
$$

where the upper bound $\omega_{b}$ is the energy scale of the coreelectron excitation energies (see Appendix F), and

$$
\epsilon(\mathbf{q}, \omega)=1+V_{\mathbf{q}} \chi^{(0)}(\mathbf{q}, \omega),
$$

with $\chi_{0}(\mathbf{q}, \omega)$ defined in Eq. (4.1.2) of Ref. [4]. Without loss of generality, we can define the tensor $K_{i, j}(\mathbf{q}, \omega)$ in the following way: 


$$
\chi^{(0)}(\mathbf{q}, \omega) \equiv \frac{2 \epsilon_{0}}{e^{2} s} \sum_{i, j} K_{i, j}(\mathbf{q}, \omega) q_{i} q_{j}
$$

In a $2 \mathrm{D}$ system, this becomes simply

$$
\chi^{(0)}(q, \omega)=\frac{2 \epsilon_{0}}{e^{2} s} K(q, \omega) q^{2},
$$

where $K(q, \omega)$ is the in-plane component [4], assumed here to be isotropic within the plane. Allowing for finite tunneling between the layers does not change $V_{q}$ [given by Eq. (F5)] as long as we stick to the model of the $\delta$ layers, but it introduces a nonzero $k_{z}$ dispersion in the singleparticle dispersion with the property $\epsilon_{k, k_{z}+2 \pi / s}=\epsilon_{k, k_{z}}$; consequently, $\chi^{(0)}$ should be a $2 \pi / s$ periodic function of $q_{z}$. Since $\chi^{(0)}$ should vanish as $q^{2}+q_{z}^{2}$ for small momentum, it is described by a series in powers of $\tilde{q}_{z}^{2 n}$, with $\tilde{q}_{z} s / 2=\sin \left(q_{z} s / 2\right)$ and $n$ an integer number. To avoid clutter in the evaluation of the $q_{z}$ integral later in this appendix - but admittedly at the cost of loss of generality-we truncate this series at $n=1$ and obtain

$$
\chi^{(0)}\left(q, q_{z}, \omega\right)=\frac{2 \epsilon_{0}}{e^{2} s}\left[K(q, \omega) q^{2}+K_{z}(q, \omega) \tilde{q}_{z}^{2}\right] .
$$

We furthermore use the MIR Ansatz that $\epsilon(q, \omega)$ has no important dispersion, at least up to $q_{0}$, so we can remove the $q$ dependence of $K$ and $K_{z}$. In parallel to the free-carrier response, there exists a bound-charge screening described by the function $S(\mathbf{q})$, which can be considered static in the range of the plasma frequency. The corresponding boundcharge dielectric function is $\epsilon_{\mathrm{sc}}(\mathbf{q})=1+S(\mathbf{q})$, so

$$
\epsilon(\mathbf{q}, \omega)=\epsilon_{\mathrm{sc}}(\mathbf{q})+\frac{\sinh q s}{q} \frac{q^{2} K(\omega)+\tilde{q}_{z}^{2} K_{z}(\omega)}{\cosh q s-\cos q_{z} s} .
$$

If the momentum dispersion of $\epsilon_{\mathrm{sc}}(\mathbf{q})$ is not too large, the dispersion has no important consequences for the Coulomb energy estimate; we therefore simply replace $\epsilon_{\mathrm{sc}}(\mathbf{q})$ by the constant $\epsilon_{\mathrm{sc}}$. At this point, it is convenient to introduce the following shorthand notation for the free-carrier response parallel (perpendicular) to the conducting layers,

$$
2 K(\omega) / \epsilon_{\mathrm{sc}}=f s, \quad 2 K_{z}(\omega) / \epsilon_{\mathrm{sc}}=g s .
$$

We insert the expression of the dielectric function, Eq. (G7), in the one for the Coulomb energy, Eq. (G2), and obtain, after integration over $q_{z}$,

$$
E_{C}^{\mathrm{mir}}=\frac{\hbar a^{2}}{4 \pi^{2}} \operatorname{Im} \int_{0}^{\omega_{b}} d \omega \int_{0}^{q_{0} s} d x \frac{x}{1+g}\left[-1+\frac{x^{2} f / 2-g[x \operatorname{coth} x-1]}{\sqrt{\left(x \operatorname{coth} x+x^{2} f / 2+g\right)^{2}-(1+g)^{2} x^{2} / \sinh ^{2} x}}\right] .
$$

In the absence of interlayer tunneling $(g=0)$, this reduces to Eq. (5.43) of Ref. [7],

$$
\begin{aligned}
E_{C}^{\mathrm{mir}}= & \frac{\hbar a^{2}}{4 \pi^{2}} \operatorname{Im} \int_{0}^{\omega_{b}} d \omega \\
& \int_{0}^{q_{0}} d q \frac{q^{2} s f / 2}{\sqrt{1+q s f \operatorname{coth} q s+(q s f / 2)^{2}}} .
\end{aligned}
$$

Reference [4] discussed the case of a purely two-dimensional system of electrons, where the effective screening of the interconduction electron Coulomb interaction is three dimensional and therefore described by the dielectric constant $\epsilon_{\mathrm{sc}}$. This limit is described by $s \rightarrow \infty$, so coth $q s \rightarrow 1$. We rearrange the integrand to $1-(1+q s f / 2)^{-1}$ and substitute the definition [4] $s f / 2 \equiv K(\omega) / \epsilon_{\mathrm{sc}}$, with the result

$E_{C}^{\mathrm{mir}}=-\frac{\hbar a^{2}}{4 \pi^{2}} \operatorname{Im} \int_{0}^{\omega_{b}} d \omega \int_{0}^{q_{0}} d q \frac{q}{1+q K(\omega) / \epsilon_{\mathrm{sc}}}$,

where we recognize Eq. (4.1.5) of Ref. [4].

The result of Eq. (G11) is shown in Fig. 22 and compared with the extrapolation schemes of Eqs. (G9) and (14) using $s=\bar{d}=7.8 \AA, q_{0}=0.31 \AA^{-1}$, and in
Eq. (14), $d=3.2 \AA$, as well as the integral of the experimental loss function for $q \approx 0$. From this comparison, we conclude that the replacement of the "true" Coulomb energy by the MIR one, plus the extrapolation to finite momentum and the $0.31 \mathrm{~A}^{-1}$ cutoff, effectively boils down to a uniform scaling of the $q=0$ result. The result is rather insensitive to the extrapolation scheme chosen and corresponds to a scaling factor $F=0.42$.

To determine the impact of temperature dependence of the $c$-axis dielectric function, we compare the output of Eq. (G9) assuming that $\epsilon_{c}$ is independent of temperature (green curve) and that the temperature dependence is described by Eq. (G1) (orange curve). Despite somewhat exaggerated assumptions about the $c$-axis temperature dependence, the influence on the Coulomb energy is negligible in comparison to that of the $a b$-plane contribution.

\section{APPENDIX H: NORMAL STATE $T$ DEPENDENCE OF $E_{C}^{\text {iso }}$}

Quite generally, increasing the quasiparticle relaxation broadens the loss-function peak, and it causes a redshift. The loss-function integral will then exhibit a corresponding 


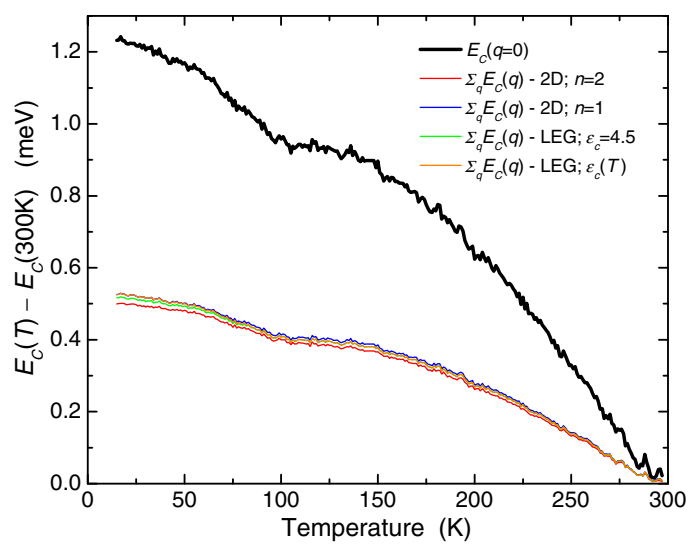

FIG. 22. Relative change of the integral of the loss function between 0 and $2.5 \mathrm{eV}$ with respect to room temperature of sample Bi2212-91-OpD. Black line: The " $q=0$ " result, same as in the lower right panel of Fig. 11. Red, blue, green, and orange curves are the finite-momentum extrapolation to finite $q_{0}=0.31 \AA^{-1}$, using different extrapolation schemes. Red curve: 2D bilayer expression, Eq. (14), with $n=2$, and $d=3.2 \AA$. Blue curve: 2D monolayer expression, Eq. (G11). Green curve: Layered electron gas (LEG) expression, Eq. (G9), with $\epsilon_{c}=\epsilon_{\mathrm{sc}}=4.5$. Orange: LEG expression, Eq. (G9), with $\epsilon_{c}(T)$ described by Eq. (G1). The overlap of orange and green curves indicates that the $c$-axis loss function has negligible influence on the temperature dependence of the Coulomb energy. The overlap of red, blue, and green curves implies that the extrapolation has negligible dependence on the type of extrapolation scheme chosen. While the black curve is the expression for the "true" Coulomb energy that one would get by simply multiplying the optically measured loss function by the area of the first Brillouin zone, the colored curves are expressions for the "MIR Coulomb energy" as discussed in Sec. IVA. The fact that the colored curves are a substantial fraction of the black one-rather than being related to it approximately by the ratio of the disk $q<0.31 \mathrm{~A}^{-1}$ to that of the first Brillouin zone-is largely attributable to the replacement of $E_{C}$ by $E_{C}^{\text {mir }}$; see Eq. (F17) for the 3D case and Eq. (G11) for the 2D case.

decrease in intensity. It is therefore of interest to see if a relation exists between the temperature dependence of the loss-function integral and the quasiparticle relaxation rate. Based on a similar reasoning, it was demonstrated in Ref. [65] that the truncation at some finite value $\Omega$ of the spectral weight integral of the optical conductivity, Eq. (8), introduces a temperature dependence of the quasiparticle relaxation rate. If indeed such a relation could be established, it would imply that in the relevant frequency range of about $1 \mathrm{eV}$, the relaxation rate at these frequencies would have the same temperature dependence for all dopings. At first glance, this appears at odds with the fact that the transport relaxation rate is known to have strong qualitatively different behavior for different doping levels. However, we cannot exclude a priori that the temperature dependence of $\gamma(\omega, T)$ is more universal among the cuprates for $\omega \sim 1 \mathrm{eV}$. Additional intensity develops when

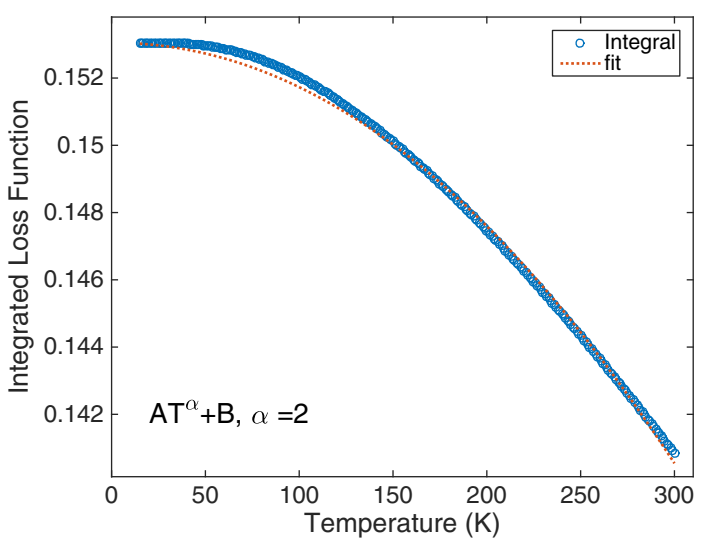

FIG. 23. Partial integral of the loss function as a function of temperature obtained by using the scattering rate in a Drude model of Norman et al. [65].

the temperature passes through the zone between $T_{n 2}$ and $T_{n 1}$, where $T_{n 2}$ is the lower bound of the region of $T^{2}$ temperature dependence, and $T_{n 1}$ is the inflection point. Aforementioned additional intensity of the integrated loss function corresponds to a gain of the partial Coulomb energy for small $q$. For the description of the dielectric properties of the interacting electrons in the normal state, we adopt the generalized Drude model

$$
\epsilon(\omega)=\epsilon_{\infty}-\frac{\omega_{p}^{2} / \omega}{\omega[1+\lambda(\omega)]+i \gamma(\omega)} .
$$

We furthermore use the model of Ref. [65] for the damping $\gamma$ and the mass renormalization constant $\lambda$. For $\omega$ larger than the energy of the fluctuations coupled to the electrons (phonons, density fluctuations) $\lambda \sim 0, \gamma$ becomes frequency independent, and its temperature dependence is

$$
\gamma(T)=2 \gamma\left[1-\frac{2 k_{B} T}{\omega_{2}} \ln \left(1-e^{-\omega_{1} / k_{B} T}\right)\right]
$$

The parameter values relevant for the present materials are $\epsilon_{\infty}=4, \gamma=0.25 \mathrm{eV}, \omega_{p}=3.1 \mathrm{eV}, \omega_{1}=15 \mathrm{meV}$, and $\omega_{2}=300 \mathrm{meV}$. The integration of the corresponding loss function is shown in Fig. 23, where the temperature dependence was extracted by a power-law fit yielding an exponent of 2 .

[1] G. V. Chester, Difference between Normal and Superconducting States of a Metal, Phys. Rev. 103, 1693 (1956).

[2] J. E. Hirsch, Apparent Violation of the Conductivity Sum Rule in Certain Superconductors, Physica (Amsterdam) 199C, 305 (1992).

[3] A. J. Leggett, A "Midinfrared" Scenario for Cuprate Superconductivity, Proc. Natl. Acad. Sci. U.S.A. 96, 8365 (1999). 
[4] A. J. Leggett, Cuprate Superconductivity: Dependence of $T_{c}$ on the c-Axis Layering Structure, Phys. Rev. Lett. 83, 392 (1999).

[5] A. J. Leggett, WHERE Is the Energy Saved in Cuprate Superconductivity?, J. Phys. Chem. Solids 59, 1729 (1998).

[6] S. Nakai, N. Nücker, H. Romberg, M. Alexander, and J. Fink, Electron Energy-Loss Studies of High- $T_{c}$ Superconductors $\mathrm{YBa}_{2} \mathrm{Cu}_{3} \mathrm{O}_{7-x}$ and $\mathrm{Bi}_{2} \mathrm{Sr}_{2} \mathrm{CaCu}_{2} \mathrm{O}_{8}$, Phys. Scr. 41, 596 (1990).

[7] C. Presura, Ph.D. thesis, University of Groningen (2003).

[8] D. van der Marel, Optical Properties of Correlated Electrons, in Strongly Correlated Systems (publisher Springer, Berlin, Heidelberg, 2015), pp. 269-296.

[9] E. Demler and S.-C. Zhang, Quantitative Test of a Microscopic Mechanism of High-Temperature Superconductivity, Nature (London) 396, 733 (1998).

[10] P. W. Anderson, Random-Phase Approximation in the Theory of Superconductivity, Phys. Rev. 112, 1900 (1958).

[11] A. J. Leggett, Theory of a Superfluid Fermi Liquid. I. General Formalism and Static Properties, Phys. Rev. 140, A1869 (1965).

[12] T. Kostyrko and R. Micnas, Collective Modes of the Extended Hubbard Model with Negative $U$ and Arbitrary Electron Density, Phys. Rev. B 46, 11025 (1992).

[13] D. van der Marel, Collective Modes of Spin, Density, Phase, and Amplitude in Exotic Superconductors, Phys. Rev. B 51, 1147 (1995).

[14] W.-C. Lee, Superconductivity-Induced Changes in Density-Density Correlation Function Enabled by Umklapp Processes, Phys. Rev. B 91, 224503 (2015).

[15] D. van der Marel (unpublished).

[16] D. J. Scalapino and S. R. White, Superconducting Condensation Energy and an Antiferromagnetic Exchange-Based Pairing Mechanism, Phys. Rev. B 58, 8222 (1998).

[17] Th. A. Maier, M. Jarrell, A. Macridin, and C. Slezak, Kinetic Energy Driven Pairing in Cuprate Superconductors, Phys. Rev. Lett. 92, 027005 (2004).

[18] K. Haule and G. Kotliar, Optical Conductivity and Kinetic Energy of the Superconducting State: A Cluster Dynamical Mean Field Study, Europhys. Lett. 77, 27007 (2007).

[19] E. Gull and A. J. Millis, Energetics of Superconductivity in the Two-Dimensional Hubbard Model, Phys. Rev. B 86, 241106 (2012).

[20] L. Fratino, P. Semon, G. Sordi, and A.-M. S. Tremblay, An Organizing Principle for Two-Dimensional Strongly Correlated Superconductivity, Sci. Rep. 6, 22715 (2016).

[21] A. J. Leggett and D. Pouliot (unpublished).

[22] This interaction should be screened by the core electrons.

[23] P. Nozières and D. Pines, Theory of Quantum Liquids (Westview Press, Boulder, 1999).

[24] P. Nozières and D. Pines, Electron Interaction in Solids. Characteristic Energy Loss Spectrum, Phys. Rev. 113, 1254 (1959).

[25] A. J. Leggett, Some Thoughts About Two Dimensionality and Cuprate Superconductivity, J. Supercond. 19, 187 (2006).

[26] P. F. Maldague, Optical Spectrum of a Hubbard Chain, Phys. Rev. B 16, 2437 (1977).

[27] D. Baeriswyl, J. Carmelo, and A. Luther, Correlation Effects on the Oscillator Strength of Optical Absorption:
Sum Rule for the One-Dimensional Hubbard Model, Phys. Rev. B 33, 7247 (1986).

[28] D. J. Scalapino, S. R. White, and S.-C. Zhang, Insulator, Metal, or Superconductor: The Criteria, Phys. Rev. B 47, 7995 (1993).

[29] D. Bergeron, V. Hankevych, B. Kyung, and A.-M.S. Tremblay, Optical and dc Conductivity of the TwoDimensional Hubbard Model in the Pseudogap Regime and Across the Antiferromagnetic Quantum Critical Point Including Vertex Corrections, Phys. Rev. B 84, 085128 (2011).

[30] H. J. A. Molegraaf, C. Presura, D. van der Marel, P. H. Kes, and M. Li, Superconductivity-Induced Transfer of In-Plane Spectral Weight in $\mathrm{Bi}_{2} \mathrm{Sr}_{2} \mathrm{CaCu}_{2} \mathrm{O}_{8+\delta}$, Science 295, 2239 (2002).

[31] P. W. Anderson, Interlayer Tunneling Mechanism for High$T_{c}$ Superconductivity: Comparison with c-Axis Infrared Experiments, Science 268, 1154 (1995).

[32] G. Deutscher, A. F. Santander-Syro, and N. Bontemps, Kinetic Energy Change with Doping upon Superfluid Condensation in High-Temperature Superconductors, Phys. Rev. B 72, 092504 (2005).

[33] F. Carbone, A. B. Kuzmenko, H. J. A. Molegraaf, E. van Heumen, V. Lukovac, F. Marsiglio, D. van der Marel, K. Haule, G. Kotliar, H. Berger, S. Courjault, P. H. Kes, and M. $\mathrm{Li}$, Doping Dependence of the Redistribution of Optical Spectral Weight in $\mathrm{Bi}_{2} \mathrm{Sr}_{2} \mathrm{CaCu}_{2} \mathrm{O}_{8+\delta}$, Phys. Rev. B 74, 064510 (2006).

[34] J. L. Tallon, C. Bernhard, H. Shaked, R. L. Hitterman, and J. D. Jorgensen, Generic Superconducting Phase Behavior in High- $T_{c}$ Cuprates: $T_{c}$ Variation with Hole Concentration in $\mathrm{YBa}_{2} \mathrm{Cu}_{3} \mathrm{O}_{7-\delta}$, Phys. Rev. B 51, 12911 (1995).

[35] M. R. Presland, J. L. Tallon, R. G. Buckley, R. S. Liu, and N. E. Flower, General Trends in Oxygen Stoichiometry Effects on $T_{c}$ in Bi and $T l$ Superconductors, Physica (Amsterdam) 176C, 95 (1991).

[36] F. R. Shapiro, The Yale Book of Quotations (publisher Yale University Press, New Haven, 2006).

[37] D. van der Marel, A. J. Leggett, J. W. Loram, and J. R. Kirtley, Condensation Energy and High- $T_{c}$ Superconductivity, Phys. Rev. B 66, 140501 (2002).

[38] A. Trokiner, L. Le Noc, J. Schneck, A. M. Pougnet, R. Mellet, J. Primot, H. Savary, Y. M. Gao, and S. Aubry, ${ }^{17} \mathrm{O}$ Nuclear-Magnetic-Resonance Evidence for Distinct Carrier Densities in the Two Types of $\mathrm{CuO}_{2}$ Planes of $(\mathrm{Bi}, \mathrm{Pb})_{2} \mathrm{Sr}_{2} \mathrm{Ca}_{2} \mathrm{Cu}_{3} \mathrm{O}_{y}$, Phys. Rev. B 44, 2426 (1991).

[39] H. Kotegawa, Y. Tokunaga, K. Ishida, G.-Q. Zheng, Y. Kitaoka, K. Asayama, H. Kito, A. Iyo, H. Ihara, K. Tanaka, K. Tokiwa, and T. Watanabe, NMR Study of Carrier Distribution and Superconductivity in Multilayered High$T_{c}$ Cuprates, J. Phys. Chem. Solids 62, 171 (2001).

[40] S. Ideta, K. Takashima, M. Hashimoto, T. Yoshida, A. Fujimori, H. Anzai, T. Fujita, Y. Nakashima, A. Ino, M. Arita, H. Namatame, M. Taniguchi, K. Ono, M. Kubota, D. H. Lu, Z.X. Shen, K. M. Kojima, and S. Uchida, Enhanced Superconducting Gaps in the Trilayer HighTemperature $\mathrm{Bi}_{2} \mathrm{Sr}_{2} \mathrm{Ca}_{2} \mathrm{Cu}_{3} \mathrm{O}_{10+\delta}$ Cuprate Superconductor, Phys. Rev. Lett. 104, 227001 (2010). 
[41] J. W. Loram, K. A. Mirza, J. R. Cooper, and W. Y. Liang, Electronic Specific Heat of $\mathrm{YBa}_{2} \mathrm{Cu}_{3} \mathrm{O}_{6+x}$ from 1.8 to 300 K, Phys. Rev. Lett. 71, 1740 (1993).

[42] J. W. Loram, K. A. Mirza, J. M. Wade, J. R. Cooper, and W. Y. Liang, The Electronic Specific Heat of Cuprate Superconductors, Physica (Amsterdam) 235-240C, 134 (1994).

[43] J. L. Tallon and J. W. Loram, The Doping Dependence of $T^{*}-$ What Is the Real High- $T_{c}$ Phase Diagram?, Physica (Amsterdam) 349C, 53 (2001).

[44] J. W. Loram, J. L. Luo, J. R. Cooper, W. Y. Liang, and J. L. Tallon, The Condensation Energy and Pseudogap Energy Scale of Bi:2212 from the Electronic Specific Heat, Physica (Amsterdam) 341-348C, 831 (2000).

[45] Th. Maier, M. Jarrell, Th. Pruschke, and M. H. Hettler, Quantum Cluster Theories, Rev. Mod. Phys. 77, 1027 (2005).

[46] V. J. Emery and S. A. Kivelson, Importance of Phase Fluctuations in Superconductors with Small Superfluid Density, Nature (London) 374, 434 (1995).

[47] Y. Wang, L. Li, M. J. Naughton, G. D. Gu, S. Uchida, and N. P. Ong, Field-Enhanced Diamagnetism in the Pseudogap State of the Cuprate $\mathrm{Bi}_{2} \mathrm{Sr}_{2} \mathrm{CaCu}_{2} \mathrm{O}_{8+\delta}$ Superconductor in an Intense Magnetic Field, Phys. Rev. Lett. 95, 247002 (2005).

[48] Y. Wang, L. Li, and N. P. Ong, Nernst Effect in High-T Superconductors, Phys. Rev. B 73, 024510 (2006).

[49] K. K. Gomes, A. N. Pasupathy, A. Pushp, S. Ono, Y. Ando, and A. Yazdani, Visualizing Pair Formation on the Atomic Scale in the High- $T_{c}$ Superconductor $\mathrm{Bi}_{2} \mathrm{Sr}_{2} \mathrm{CaCu}_{2} \mathrm{O}_{8+\delta}$, Nature (London) 447, 569 (2007).

[50] J. L. Tallon, J. G. Storey, and J. W. Loram, Fluctuations and Critical Temperature Reduction in Cuprate Superconductors, Phys. Rev. B 83, 092502 (2011).

[51] A. Dubroka, M. Rössle, K. W. Kim, V. K. Malik, D. Munzar, D. N. Basov, A. A. Schafgans, S. J. Moon, C. T. Lin, D. Haug et al., Evidence of a Precursor Superconducting Phase at Temperatures as High as $180 \mathrm{~K}$ in $\mathrm{RBa}_{2} \mathrm{Cu}_{3} \mathrm{O}_{7-\delta}(R=Y, G d, E u)$ Superconducting Crystals from Infrared Spectroscopy, Phys. Rev. Lett. 106, 047006 (2011).

[52] T. Kondo, Y. Hamaya, A. D. Palczewski, and T. Takeuchi, Disentangling Cooper-Pair Formation Above the Transition Temperature from the Pseudogap State in the Cuprates, Nat. Phys. 7, 21 (2011).

[53] E. Uykur, K. Tanaka, T. Masui, S. Miyasaka, and S. Tajima, Persistence of the Superconducting Condensate Far Above the Critical Temperature of $\mathrm{YBa}_{2}(\mathrm{Cu}, \mathrm{Zn})_{3} \mathrm{O}_{y}$ Revealed by c-Axis Optical Conductivity Measurements for Several Zn Concentrations and Carrier Doping Levels, Phys. Rev. Lett. 112, 127003 (2014).

[54] T. Kondo, W. Malaeb, Y. Ishida, T. Sasagawa, H. Sakamoto, T. Takeuchi, T. Tohyama, and S. Shin, Point Nodes Persisting Far Beyond $T_{c}$ in Bi2212, Nat. Commun. 6, 7699 (2015).

[55] O. Cyr-Choinière, D. LeBoeuf, S. Badoux, S. DufourBeauséjour, D. A. Bonn, W. N. Hardy, R. Liang, N. Doiron-Leyraud, and L. Taillefer, Suppression of Charge Order by Pressure in the Cuprate Superconductor
$\mathrm{YBa}_{2} \mathrm{Cu}_{3} \mathrm{O}_{y}$ : Restoring the Full Superconducting Dome, arXiv: $1503.02033 \mathrm{v} 1$.

[56] Y. Sidis and P. Bourges, Evidence for Intra-Unit-Cell Magnetic Order in the Pseudo-gap State of High- $T_{c}$ Cuprates, J. Phys. Conf. Ser. 449, 012012 (2013).

[57] T. Wu, H. Mayaffre, S. Krämer, M. Horvatić, C. Berthier, W. N. Hardy, R. Liang, D. A. Bonn, and M.-H. Julien, Magnetic-Field-Induced Charge-Stripe Order in the HighTemperature Superconductor $\mathrm{YBa}_{2} \mathrm{Cu}_{3} \mathrm{O}_{y}, \quad$ Nature (London) 477, 191 (2011).

[58] T. Wu, H. Mayaffre, S. Krämer, M. Horvatić, C. Berthier, P. L. Kuhns, A. P. Reyes, R. Liang, W. N. Hardy, D. A. Bonn, and M.-H. Julien, Emergence of Charge Order from the Vortex State of a High-Temperature Superconductor, Nat. Commun. 4, 2113 (2013).

[59] M. Hashimoto, R.-H. He, K. Tanaka, J. P. Testaud, W. Meevasana, R. G. Moore, D. H. Lu, H. Yao, Y. Yoshida, H. Eisaki, T.P. Devereaux, Z. Hussain, and Z.-X. Shen, Particle-Hole Symmetry Breaking in the Pseudogap State of Bi2201, Nat. Phys. 6, 414 (2010).

[60] S. Blanco-Canosa, A. Frano, E. Schierle, J. Porras, T. Loew, M. Minola, M. Bluschke, E. Weschke, B. Keimer, and M. Le Tacon, Resonant X-Ray Scattering Study of ChargeDensity Wave Correlations in $\mathrm{YBa}_{2} \mathrm{Cu}_{3} \mathrm{O}_{6+x}$, Phys. Rev. B 90, 054513 (2014).

[61] M. Hücker, N. B. Christensen, A. T. Holmes, E. Blackburn, E. M. Forgan, R. Liang, D. A. Bonn, W. N. Hardy, O. Gutowski, M. von Zimmermann, S. M. Hayden, and J. Chang, Competing Charge, Spin, and Superconducting Orders in Underdoped $\mathrm{YBa}_{2} \mathrm{Cu}_{3} \mathrm{O}_{y}$, Phys. Rev. B 90, 054514 (2014).

[62] T. P. Croft, C. Lester, M. S. Senn, A. Bombardi, and S. M. Hayden, Charge Density Wave Fluctuations in $\mathrm{La}_{2-x} \mathrm{Sr}_{x} \mathrm{CuO}_{4}$ and Their Competition with Superconductivity, Phys. Rev. B 89, 224513 (2014).

[63] M. J. Lawler, K. Fujita, J. Lee, A. R. Schmidt, Y. Kohsaka, C. K. Kim, H. Eisaki, S. Uchida, J. C. Davis, J. P. Sethna, and E.-A. Kim, Intra-Unit-Cell Electronic Nematicity of the High-T ${ }_{c}$ Copper-Oxide Pseudogap States, Nature (London) 466, 347 (2010).

[64] C. V. Parker, P. Aynajian, E. H. da Silva Neto, A. Pushp, J. Ono, J. Wen, Z. Xu, G. D. Gu, and A. Yazdani, Fluctuating Stripes at the Onset of the Pseudogap in the High- $T_{c}$ Superconductor $\mathrm{Bi}_{2} \mathrm{Sr}_{2} \mathrm{CaCu}_{2} \mathrm{O}_{8+x}$, Nature (London) 468, 677 (2010).

[65] M. R. Norman, A. V. Chubukov, E. van Heumen, A. B. Kuzmenko, and D. van der Marel, Optical Integral in the Cuprates and the Question of Sum-Rule Violation, Phys. Rev. B 76, 220509 (2007).

[66] The slight undershoot of the data as compared to the fitted power law appears to vanish gradually as a function of increasing temperature. Consequently, the fitted curve and the experimental data appear to merge at a temperature determined by the instrument noise, which obviously has no particular significance with respect to the state of matter of the material.

[67] E. Giannini, V. Garnier, R. Gladyshevskii, and R. Flükiger, Growth and Characterization of $\mathrm{Bi}_{2} \mathrm{Sr}_{2} \mathrm{Ca}_{2} \mathrm{Cu}_{3} \mathrm{O}_{10}$ and $(\mathrm{Bi}, \mathrm{Pb})_{2} \mathrm{Sr}_{2} \mathrm{Ca}_{2} \mathrm{Cu}_{3} \mathrm{O}_{10-\delta}$ Single Crystals, Supercond. Sci. Technol. 17, 220 (2004). 
[68] J. S. Wen, Z. J. Xu, G. Y. Xu, M. Hücker, J. M. Tranquada, and G. D. Gu, Large Bi-2212 Single Crystal Growth by the Floating-Zone Technique, J. Cryst. Growth 310, 1401 (2008).

[69] D. E. Aspnes, Approximate Solution of Ellipsometric Equations for Optically Biaxial Crystals, J. Opt. Soc. Am. 70, 1275 (1980).

[70] S. Tajima, G. D. Gu, S. Miyamoto, A. Odagawa, and N. Koshizuka, Optical Evidence for Strong Anisotropy in the Normal and Superconducting States in $\mathrm{Bi}_{2} \mathrm{Sr}_{2} \mathrm{CaCu}_{2} \mathrm{O}_{8+z}$, Phys. Rev. B 48, 16164 (1993).

[71] N. Petit, V. Garnier, V. T. Phuoc, R. Caillard, A. M. Frelin, A. Ruyter, I. Laffez, J. C. Soret, A. Maignan, and F. Gervais, Polarized Infrared Reflectivity Study of an Oriented Ceramic of $\mathrm{Bi}_{2} \mathrm{Sr}_{2} \mathrm{Ca}_{2} \mathrm{Cu}_{3} \mathrm{O}_{10+\delta}$ (Bi-2223), Eur. Phys. J. B 25, 423 (2002).

[72] A. J. Leggett, Interlayer Tunneling Models of Cuprate Superconductivity: Implications of a Recent Experiment, Science 274, 587 (1996).

[73] A. A. Tsvetkov, D. van der Marel, K. A. Moler, J. R. Kirtley, J. L. de Boer, A. Meetsma, Z. F. Ren, N. Koleshnikov, D. Dulic, A. Damascelli et al., Global and Local Measures of the Intrinsic Josephson Coupling in $\mathrm{Tl}_{2} \mathrm{Ba}_{2} \mathrm{CuO}_{6}$ as a Test of the Interlayer Tunnelling Model, Nature (London) 395, 360 (1998).

[74] D. N. Basov, S. I. Woods, A. S. Katz, E. J. Singley, R. C. Dynes, M. Xu, D. G. Hinks, C. C. Homes, and M. Strongin, Sum Rules and Interlayer Conductivity of High- $T_{c}$ Cuprates, Science 283, 49 (1999).

[75] D. van der Marel and A. Tsvetkov, Transverse Optical Plasmons in Layered Superconductors, Czech. J. Phys. 46, 3165 (1996).

[76] V. Železný, S. Tajima, D. Munzar, T. Motohashi, J. Shimoyama, and K. Kishio, Anomalies in the Infrared Spectra of Underdoped $\mathrm{Bi}_{2} \mathrm{Sr}_{2} \mathrm{CaCu}_{2} \mathrm{O}_{z}$ as Evidence for the Intrabilayer Josephson Effect, Phys. Rev. B 63, 060502 (2001).

[77] F. Carbone, A. B. Kuz'menko, H. Molegraaf, E. van Heumen, E. Giannini, and D. van der Marel, In-Plane Optical Spectral Weight Transfer in Optimally Doped $\mathrm{Bi}_{2} \mathrm{Sr}_{2} \mathrm{Ca}_{2} \mathrm{Cu}_{3} \mathrm{O}_{10}$, Phys. Rev. B 74, 024502 (2006).

[78] A. L. Fetter, Electrodynamics of a Layered Electron Gas. II. Periodic Array, Ann. Phys. (N.Y.) 88, 1 (1974).

[79] H. Morawitz, I. Bozovic, V. Z. Kresin, G. Rietveld, and D. van der Marel, The Plasmon Density of States of a Layered Electron Gas, Z. Phys. B 90, 277 (1993). 